\title{
Fick diffusion coefficients via Molecular Dynamics: an alternative approach in the Fourier domain
}

\author{
Thiago J. P. dos Santos, ${ }^{\dagger}$ Frederico W. Tavares,,$+\dagger$ and Charlles R. A. Abreu, ${ }^{*, \ddagger}$ \\ $\dagger$ Chemical Engineering Program - COPPE, Federal University of Rio de Janeiro, Rio de \\ Janeiro 68542, Brazil \\ $\ddagger$ School of Chemistry, Federal University of Rio de Janeiro, Rio de Janeiro 68542, Brazil \\ E-mail: abreu@eq.ufrj.br
}

\begin{abstract}
Mutual diffusion coefficient data are required for several systems of scientific and engineering interest to properly describe mass transport phenomena over a wide range of pressures, temperatures, and compositions. In this work, we calculated Fick diffusion coefficients for some $\mathrm{CO}_{2}+$ n-alkane mixtures at high pressures using a new method, which we derived by introducing modifications to the Fourier Correlation Method (FCM) originally proposed by Nichols and Wheeler [I\&EC Research, 54, 12156-12164 (2015)]. The modified FCM (mFCM) results were validated through comparisons with experimental data and with Fick coefficients calculated by employing well-established Molecular Dynamics methodologies. The new approach has some interesting advantages, such as providing Fick coefficients for molecular systems directly through a single equilibrium calculation, in contrast to traditional methods in which an extra calculation is needed to obtain the so-called thermodynamic factor. It is shown that the new approach considerably reduces the finite-size effect of the simulation box on the calculated
\end{abstract}


diffusion coefficients, which are thus obtained in the thermodynamic limit.

\section{Introduction}

Mass transfer phenomena are crucial for the development and improvement of technologies in several areas, from biomedical and biotechnology applications to the oil and gas industries. ${ }^{1}$ In the chemical industry, mass transfer processes are quite common and usually mass diffusion phenomena are not only the heart of an operation, but also its limiting step. Therefore, the appropriate description of the mutual diffusion process is critical for industrial equipment and process design. 2

It is important to distinguish mutual diffusion from self-diffusion. Self-diffusion is an equilibrium phenomenon consisting of molecular diffusion due to collisions and Brownian motion, in which the net mass flow of each component is zero ${ }^{3 / 4}$ On the other hand, mutual diffusion is a non-equilibrium process that takes place due to the presence of a chemical potential gradient in the medium, causing a non-zero mass flow of each component and increasing the entropy of the system as it moves to equilibrium. ${ }^{5}$

In analogy to Fourier's thermal conduction law, Fick proposed an equation to the molar flow $\boldsymbol{J}_{\mathbf{1}}$ of a given species 1 in a binary mixture by stating that this quantity must be directly proportional to the concentration difference of this species and inversely proportional to the distance corresponding to this difference. ${ }^{[6] 7}$ In the infinitesimal limit and for the case of a multi-component mixture, the generalized Fick's law can be applied to determine the $\left(n_{c}-1\right)$ independent diffusion flows according to the equation ${ }^{8}$

$$
\boldsymbol{J}_{\boldsymbol{i}}=-c_{t} \sum_{j=1}^{n_{c}-1} D_{i j} \nabla x_{j}
$$

where $\sum_{i=1}^{n_{c}} \boldsymbol{J}_{\boldsymbol{i}}=0, c_{t}$ is the total molar concentration, and $\nabla x_{i}$ is the mole fraction gradient of species $i$ in the mixture. The coefficients $D_{i j}$ are a generalized kind of Fick coefficients, which does not depend on the magnitude of the concentration gradient. ${ }^{7}$ Unlike the binary 
Fick diffusivities, the generalized Fickian coefficients depend on the definition of the reference velocity frame, do not directly reflect the $i$ - $j$ interactions, can either assume positive or negative values, and are not necessarily symmetrical $\left(D_{i j} \neq D_{j i}\right)$. [8

A different approach for describing mutual diffusion is through the so-called MaxwellStefan (MS) equations. $\frac{9110}{10}$ The MS approach considers that diffusional flow is the result of a balance between molecular friction forces and intermolecular interactions, $\frac{11112}{1}$ correlating the flows directly with the motive force of chemical potential gradient through the equation

$$
\frac{x_{i}}{R T} \nabla_{T, P} \mu_{i}=\sum_{\substack{j=1 \\ j \neq i}}^{n_{c}} \frac{\left(x_{i} \boldsymbol{J}_{j}-x_{j} \boldsymbol{J}_{\boldsymbol{i}}\right)}{c_{t} \mathcal{D}_{i j}},
$$

where $R$ is the universal gas constant, $T$ is the absolute temperature, $\mu_{i}$ is the chemical potential of component $i$, and $\mathcal{D}_{i j}$ are the so-called MS coefficients. Unlike Fick diffusion coefficients for the multicomponent case, MS coefficients are independent of the reference velocity frame, have a clearer physical interpretation, and are always positive and symmetrical $\left(\mathcal{D}_{i j}=\mathcal{D}_{j i}\right) .{ }^{8}$ Nevertheless, MS coefficients normally cannot be directly measured by experimental means, since they are formal coefficients that do not take into account the non-ideal thermodynamic effects of a mixture. This justifies the lower dependence of the MS coefficients on the composition of the mixture. ${ }^{8}$

Although they have different constructions, both Fick and MS formalisms describe the same physical phenomenon and must therefore have some equivalence. In a molar reference frame and for a two-component system, it is valid that 13

$$
D_{12}=\Gamma \mathcal{D}_{12}
$$

where $\Gamma$ is the so-called thermodynamic factor for a binary mixture, given by

$$
\Gamma=1+x_{1}\left(\frac{\partial \ln \gamma_{1}}{\partial x_{1}}\right)_{T, P, N_{2}}
$$


where $\gamma_{1}$ is the activity coefficient of component 1 in the mixture, and the subscripts $T, P$, and $N_{2}$ indicate that the partial differentiation is carried out at constant temperature, pressure, and number of molecules of component 2. ${ }^{14}$ It is noteworthy that the thermodynamic factor plays the role of introducing the non-ideal effects of the mixture into the MS coefficient, and for this reason the Fick coefficient is usually more strongly dependent on the composition of the system. The generalized form of the relation between Fick and MS coefficients for the multicomponent case is given in a matrix form and can be found in several mass-transfer classical references. $\frac{8115}{2}$

It is also worth noting that both Fick and MS coefficients are not only dependent on the composition, but also on the temperature and pressure of the medium. This represents a serious challenge for engineers, because industrial processes take place in large ranges of temperatures, pressures, and concentrations, but experimental measurements of ordinary diffusion coefficients in each scenario of interest can be unfeasible. As alternatives to the experiments, there exist several approaches to estimate mutual diffusion coefficients, including semi-empirical correlations and the so-called Darken relations. $\frac{16}{\text { Most }}$ of these relations make mutual diffusivity a function of composition, temperature, self-diffusivity, and MS coefficients at infinite dilution, which can be obtained experimentally. ${ }^{14}$ Another possibility for obtaining ordinary diffusion coefficients for systems of industrial interest is through molecular simulations. Although they are not normally seen as practical engineering tool yet, molecular simulations are already widely used for the calculation of mass transport properties, including MS and Fick diffusivities. $17717 \sqrt[23]{23}$

In this work, we calculated Fick diffusion coefficients of several $\mathrm{CO}_{2}+$ n-alkane mixtures at high pressures using a variation of the methodology proposed by Nichols and Wheeler. ${ }^{22}$ We called this new approach as the modified Fourier Correlation Method (mFCM). Because the original Fourier Correlation Method was previously validated for LJ-fluid mixtures only and proved to be not consistent for more complex fluids, some modifications were needed in order to generalize it to the case of molecular systems. For further validation of the mFCM, we 
also compared the obtained results with experimental data and with diffusivities calculated through the traditional approach based on Onsager coefficients for the determination of the MS coefficients, followed by conversion to Fick diffusivities by using thermodynamic factors. These are also calculated in a Fourier-domain approach, based on the KirkwoodBuff formalism. Finally, we were able to evaluate how the simulation box size influences Fick coefficients determined through the $\mathrm{mFCM}$, which proved to be practically immune to the finite-size of the simulation box.

\section{Theoretical Background}

\section{Fick Diffusion Coefficients via Molecular Dynamics}

In the context of Molecular Dynamics, there are several systematic ways of calculating mutual diffusion coefficients. One possible way is to impose a mass flow in the simulation box and determine the mutual diffusion coefficient by measuring the established composition gradient in the box, according to Eq. (1). This approach can be achieved by using Reverse Non-Equilibrium Molecular Dynamics methods (R-NEMD), by exchanging positions and velocity vectors of molecules in different regions of the simulation box to force the occurrence of a constant composition gradient. $\frac{19}{19}$ Although somewhat intuitive, this methodology presents some problems such as spurious energy drifts caused by the R-NEMD algorithms, high gradient magnitudes and fluctuations, and the fact that the results obtained so far are only qualitative. 1920

In general, molecular simulation approaches for calculating diffusion coefficients employ Equilibrium Molecular Dynamics (EMD) methods, by analyzing the equilibrium fluctuations. Besides avoiding spurious problems of non-equilibrium methodologies, EMD approaches also enables other properties to be calculated in the same simulation. Commonly, equilibrium simulations provide us with Maxwell-Stefan coefficients, which can be converted into Fick diffusion coefficients by multiplying the thermodynamic factor, according to Eq. (3).21 
A possible way to determine MS diffusivities in equilibrium simulations is through the extended Einstein relation for mutual diffusion. 18 Another possible approach is through the equivalent Green-Kubo formalism. $\frac{18 \mid 23124}{1 n}$ theory, both Einstein and Green-Kubo formalisms for mutual diffusion should lead to similar results, although neither approach is usually applied for the computation of such coefficient. Actually, one of the most widely used methodologies for the computation of MS coefficients is based on the Onsager formalism of irreversible thermodynamics. $\frac{25}{25}$ This approach starts with the calculation of an $n_{c^{-}}$dimensional matrix of the so-called Onsager coefficients, whose entries $\Lambda_{i j}$ are given by 17

$$
\Lambda_{i j}=-\lim _{t \rightarrow \infty}\left\{\frac{1}{6 t} \frac{1}{N}\left\langle\left(\sum_{l=1}^{N_{i}}\left[\boldsymbol{r}_{l, i}(t)-\boldsymbol{r}_{l, i}(0)\right]\right) \cdot\left(\sum_{k=1}^{N_{j}}\left[\boldsymbol{r}_{\boldsymbol{k}, \boldsymbol{j}}(t)-\boldsymbol{r}_{\boldsymbol{k}, \boldsymbol{j}}(0)\right]\right)\right\rangle\right\}
$$

where the indexes $l$ and $k$ represent a sum over the center-of-mass position of each molecule of types $i$ and $j$, respectively. Note that Eq. (5) is in accordance with the Onsager reciprocal relations, which imply that these coefficients are symmetric $\left(\Lambda_{i j}=\Lambda_{j i}\right)$. For binary mixtures, the Onsager coefficients are correlated to the MS diffusivities through the relation 14

$$
\mathcal{D}_{12}=\frac{x_{2}}{x_{1}} \Lambda_{11}+\frac{x_{1}}{x_{2}} \Lambda_{22}-2 \Lambda_{12}
$$

The generalization of the Eq. (6) for an $n_{c}$-component mixture in matrix notation can be found in many references. $\frac{714 \mid 17}{}$ Despite this approach being quite efficient, its main difficulty lies on the calculation of the thermodynamic factor to convert MS coefficients into Fick diffusivities. Among the strategies to perform such calculation, those who adopt the Kirkwood-Buff (KB) formalism in EMD simulations are widely employed. However, these approaches are highly susceptible to finite-size effects of the simulation box, and KB-integrals may not converge even for simple molecular systems. $\frac{26 \mid 27}{27}$ There are several recent attempts in the literature to increase the convergence of such integrals for the calculation of the socalled KB coefficients and of thermodynamic factors, $\frac{27}{29}$ including alternative strategies in the Fourier domain. $\stackrel{30}{ }$ Despite all the development, this is still a field of active research in 
the literature.

\section{The Fourier Correlation Method (FCM)}

Recently, Nichols and Wheeler ${ }^{22}$ proposed the FCM for the direct calculation of Fick diffusion coefficients of binary mixtures through simulations at equilibrium. Based on the mathematical device of correlations in the Fourier domain applied to Fick's Second Law of diffusion, they derived a governing diffusion equation that proved to be very useful in Molecular Dynamics simulations. This allows the calculation of Fick coefficients in the thermodynamic limit condition and, therefore, supposedly devoid of the finite-size effect of the simulation box. They initially defined a complex factor of component $i$, which can be calculated from the molecule positions over time through the relation 22

$$
\psi_{i}(\boldsymbol{q}, t)=\frac{1}{N} \sum_{l=1}^{N_{i}} e^{-\hat{i} \boldsymbol{q} \cdot \boldsymbol{r}_{l, i}(t)}
$$

where $N$ is the total number of molecules in the system, $N_{i}$ is the number of molecules of type $i, \hat{i}$ is the imaginary number, and $\boldsymbol{q}$ is the wave or lattice vector in the reciprocal Fourier space, given by

$$
\boldsymbol{q}=\frac{2 \pi}{L}\left[\begin{array}{l}
m_{x} \\
m_{y} \\
m_{z}
\end{array}\right]
$$

in which $L$ is the edge length of the cubic box, and the components $m_{x}, m_{y}, m_{z}$ are three independent integers $\left(\boldsymbol{m} \in \mathbb{Z}^{3}\right)$. Thus, they employed Equation (7) as an strategy to introduce a finite spatial Fourier transform into the Fick's Second Law and turn the problem into a simple ordinary differential equation (ODE), after performing an analysis of the diffusion equation based on the Linear Response Theory. Then, they introduced a self-correlation expression by multiplying both sides of the solution by the complex conjugate of $N \psi_{i}(\boldsymbol{q}, 0)$, 
resulting in ${ }^{222}$

$$
S_{i i}(\boldsymbol{q}, t)=S_{i i}(\boldsymbol{q}, 0) \mathrm{e}^{-D_{12}(\boldsymbol{q}) q^{2} t},
$$

where $q=|\boldsymbol{q}|$ and $S_{i i}(\boldsymbol{q}, t)$ is a dynamic factor, which contains information about the time evolution of the particle self-correlations, and is expressed by

$$
S_{i i}(\boldsymbol{q}, t)=N\left\langle\psi_{i}(\boldsymbol{q}, t) \psi_{i}(-\boldsymbol{q}, 0)\right\rangle .
$$

A similar expression as in Eq. (9) can be found in classical texts. ${ }^{31}$ It is important to emphasise that this approach directly provides Fick diffusivities because it implicitly includes the thermodynamic factor calculation. In a previous work, Nichols, Moore, and Wheeler ${ }^{30}$ showed that this factor can also be calculated in the Fourier domain based on the KirkwoodBuff formalism, according to the equation ${ }^{30132}$

$$
\Gamma(\boldsymbol{q})=\frac{N_{i} N_{j}}{N_{j}^{2} S_{i i}(\boldsymbol{q})-2 N_{i} N_{j} S_{i j}(\boldsymbol{q})+N_{i}^{2} S_{j j}(\boldsymbol{q})},
$$

in which $S_{i j}(\boldsymbol{q})$ is the well-known static structure factor, given by

$$
S_{i j}(\boldsymbol{q})=N\left\langle\psi_{i}(\boldsymbol{q}) \psi_{j}(-\boldsymbol{q})\right\rangle .
$$

Finally, based on Eq. (9), the mutual diffusion coefficient for each lattice vector can be determined by a linear regression through the relation

$$
D_{12}(\boldsymbol{q}) t=-\frac{1}{q^{2}} \ln \left[\frac{S_{i i}(\boldsymbol{q}, t)}{S_{i i}(\boldsymbol{q}, 0)}\right]=\phi_{i i}
$$

where the diffusion coefficient in the thermodynamic limit can be determined by extrapolating the $D_{12}(\boldsymbol{q})$ results to $q \rightarrow 0$, which is equivalent to determining the diffusivity in a macroscopic simulation box $(L \rightarrow \infty)$.

In their work, Nichols and Wheeler ${ }^{221}$ adopted the strategy of averaging the results ob- 
tained for both components 1 and 2 through Eq. (13), that is, an average between $D_{12}$ obtained from $\phi_{11}(\boldsymbol{q}, t)$ and $\phi_{22}(\boldsymbol{q}, t)$. Besides, they validated the FCM approach for LennardJones binary fluids at ordinary temperatures and pressures, and employed an empirical correlation to extrapolate the results to the thermodynamic limit. However, there is no evidence that this approach could work for binary mixtures more complex than Lennard-Jones fluids. In addition, there is no extension of this methodology to the computation of mutual diffusion coefficients of molecular systems.

\section{Methodology}

\section{The modified Fourier Correlation Method (mFCM)}

Here we present our proposed modifications to the original FCM construction, mainly involving the governing diffusion equation of the method. In a binary mixture, for all $\boldsymbol{q} \neq \mathbf{0}$, the linear regime ensures that ${ }^{22}$

$$
\frac{d \psi_{i}(\boldsymbol{q}, t)}{d t}=-D_{12}(q) q^{2} \psi_{i}(\boldsymbol{q}, t)
$$

One way of making this equation useful for computing $D_{12}(q)$ from EMD trajectories is to multiply both sides by a constant $N \psi_{j}(-\boldsymbol{q}, 0)$ and take a canonical average. This results in

$$
\frac{d S_{i j}(\boldsymbol{q}, t)}{d t}=-D_{12}(q) q^{2} S_{i j}(\boldsymbol{q}, t)
$$

which is similar to the differential equation whose solution is given by Eq. (9). In Eq. (15), $S_{i j}(\boldsymbol{q}, t)$ is a dynamic cross-correlation factor, given by

$$
S_{i j}(\boldsymbol{q}, t)=N\left\langle\psi_{i}(\boldsymbol{q}, t) \psi_{j}(-\boldsymbol{q}, 0)\right\rangle=\frac{1}{N}\left\langle\left(\sum_{l=1}^{N_{i}} e^{-\hat{i} \boldsymbol{q} \cdot \boldsymbol{r}_{l, i}(t)}\right)\left(\sum_{k=1}^{N_{j}} e^{\hat{i} \boldsymbol{q} \cdot \boldsymbol{r}_{k, j}(0)}\right)\right\rangle .
$$


Therefore, $S_{i j}(\boldsymbol{q}, t)$ is a time-correlation function that can be estimated from a MD trajectory. Contrary to what is stated in Ref. 22, $S_{i j}(\boldsymbol{q}, t)$ is not a real-valued function. However, when an average is taken for all $q$ vectors with equal length, the imaginary parts cancel out. Thus, since $S_{i j}(-\boldsymbol{q}, t)$ is the complex conjugate of $S_{i j}(\boldsymbol{q}, t)$, we can deal exclusively with its real part by defining

$$
\mathbb{S}_{i j}(\boldsymbol{q}, t)=\frac{S_{i j}(\boldsymbol{q}, t)+S_{i j}(-\boldsymbol{q}, t)}{2},
$$

which is equivalent to

$$
\begin{aligned}
\mathbb{S}_{i j}(\boldsymbol{q}, t)=\frac{1}{N}\left\langle\left[\sum_{l=1}^{N_{i}} \cos \left(\boldsymbol{q} \cdot \boldsymbol{r}_{l, i}(t)\right)\right]\left[\sum_{k=1}^{N_{j}} \cos \left(\boldsymbol{q} \cdot \boldsymbol{r}_{k, j}(0)\right)\right]+\right. & \\
& {\left.\left[\sum_{l=1}^{N_{i}} \sin \left(\boldsymbol{q} \cdot \boldsymbol{r}_{l, i}(t)\right)\right]\left[\sum_{k=1}^{N_{j}} \sin \left(\boldsymbol{q} \cdot \boldsymbol{r}_{k, j}(0)\right)\right]\right\rangle . }
\end{aligned}
$$

Note that, for a binary system, we can define a more general quantity $\mathbb{S}(\boldsymbol{q}, t)$, given by

$$
\mathbb{S}(\boldsymbol{q}, t)=\sum_{i=1}^{n_{c}} \sum_{j=1}^{n_{c}} \mathbb{S}_{i j}(\boldsymbol{q}, t)=\mathbb{S}_{11}(\boldsymbol{q}, t)+\mathbb{S}_{12}(\boldsymbol{q}, t)+\mathbb{S}_{21}(\boldsymbol{q}, t)+\mathbb{S}_{22}(\boldsymbol{q}, t),
$$

which is the real part of the well-known intermediate scattering function. ${ }^{31}$ Thus, we can substitute the general quantity defined in Eq. (19) into Eq. (15). Considering a given wave number $q$, since $\|\boldsymbol{q}\|=\|-\boldsymbol{q}\|=q$, it follows that

$$
D_{12}(\boldsymbol{q})=-\frac{1}{q^{2}} \frac{\dot{\mathbb{S}}(\boldsymbol{q}, t)}{\mathbb{S}(\boldsymbol{q}, t)}
$$

There are, at least, two possible ways of evaluating the ratio above. The first one consists in evaluating both $\mathbb{S}(\boldsymbol{q}, t)$ and $\dot{\mathbb{S}}(\boldsymbol{q}, t)$ from ensemble averages. By taking the time-derivative of Eq. (19) and considering Eq. (18), we have

$$
\dot{\mathbb{S}}(\boldsymbol{q}, t)=\sum_{i=1}^{n_{c}} \sum_{j=1}^{n_{c}} \frac{1}{N}\left\langle\sum_{l=1}^{N_{i}} \sum_{k=1}^{N_{j}} \sin \left(\boldsymbol{q} \cdot\left[\boldsymbol{r}_{l, i}(t)-\boldsymbol{r}_{k, j}(0)\right]\right) \boldsymbol{q} \cdot \boldsymbol{v}_{l, i}(t)\right\rangle .
$$


The second way consists in plotting the equation resulting from the ODE resolution, that is

$$
D_{12}(q) t=-\frac{1}{q^{2}} \ln \left[\frac{\mathbb{S}(\boldsymbol{q}, t)}{\mathbb{S}(\boldsymbol{q}, 0)}\right]
$$

and taking the angular coefficient of its linear region, which is similar to Eq. (13).

It is worth noting that $\mathbb{S}(\boldsymbol{q}, t)$ is the spatial Fourier transform of the van Hove paircorrelation function $G(\boldsymbol{r}, t)$, and thus can be split into two separated contributions ${ }^{31}$

$$
\mathbb{S}(\boldsymbol{q}, t)=\mathbb{S}_{S}(\boldsymbol{q}, t)+\mathbb{S}_{D}(\boldsymbol{q}, t)
$$

where $\mathbb{S}_{S}(\boldsymbol{q}, t)$ is the real part of the self-intermediate scattering function, given by

$$
\mathbb{S}_{S}(\boldsymbol{q}, t)=\mathbb{S}_{11}(\boldsymbol{q}, t)+\mathbb{S}_{22}(\boldsymbol{q}, t)
$$

and $\mathbb{S}_{D}(\boldsymbol{q}, t)$ is the real part of the distinct-intermediate scattering function,

$$
\mathbb{S}_{D}(\boldsymbol{q}, t)=\mathbb{S}_{12}(\boldsymbol{q}, t)+\mathbb{S}_{21}(\boldsymbol{q}, t)
$$

It is important to clarify that $\mathbb{S}_{S}(\boldsymbol{q}, t)$ and $\mathbb{S}_{D}(\boldsymbol{q}, t)$ present different physical behaviours, since as $t \rightarrow \infty$ the function $G_{S}(\boldsymbol{r}, t)$ tends to a bell-shaped curve, whereas the peaks of $G_{D}(\boldsymbol{r}, t)$ tend to decay and disappear. 31

Note that the original Fourier Correlation Method ${ }^{22}$ is equivalent to compute $D_{12}(q)$ by considering the real terms of the self-intermediate scattering function $\mathbb{S}_{S}(\boldsymbol{q}, t)$ separately and then average the results obtained by employing $\mathbb{S}_{11}$ and $\mathbb{S}_{22}$. However, we observed that the self-intermediate scattering function is not appropriate for the calculation of diffusivities in all types of mixtures, mainly if the two components in the system have different molecular structures (see the Results and Discussion section). In such cases, each component provides a specific self-intermediate scattering function, and this may yield two different diffusivity 
results. This will be discussed in the forthcoming sections.

Our new proposal involves replacing the originally employed self-intermediate scattering function by the distinct-intermediate scattering function $\mathbb{S}_{D}(\boldsymbol{q}, t)$. Thus, the main equation of the mFCM is given by

$$
D_{12}(q) t=-\frac{1}{q^{2}} \ln \left[\frac{\mathbb{S}_{D}(\boldsymbol{q}, t)}{\mathbb{S}_{D}(\boldsymbol{q}, 0)}\right]=\phi_{D}
$$

whose results proved to be entirely consistent and free of disparities. The final diffusivities are obtained by extrapolating the $D_{12}(q)$ results to the limit of $q \rightarrow 0$. Note that the determination of $D_{12}(0)$ in the Fourier Domain is equivalent to calculating the mutual diffusion coefficient in the real space with an infinite simulation box $(L \rightarrow \infty)$. Thus, the extrapolated Fick coefficients are expected to be devoid of finite-size effects. The modified approach provides results in accordance with experimental data, even for binary systems more complex than LJ-mixtures.

\section{Simulation details}

We have studied $\mathrm{CO}_{2}$ at infinite dilution in either n-hexane or n-octane under pressures ranging from 1.0 to $68.0 \mathrm{MPa}$ and temperatures of $298 \mathrm{~K}, 323 \mathrm{~K}$, and $373 \mathrm{~K}$. We also studied $\mathrm{CO}_{2}+$ n-octane mixtures at $290 \mathrm{~K}$ and $311 \mathrm{~K}$, under pressures ranging from 0.75 to $3.68 \mathrm{MPa}$, with their corresponding bubble-point concentrations of carbon dioxide. We employed in the simulations the OPLS (united atoms) ${ }^{33}$ force field for the hydrocarbons and the single-site model proposed by Higashi et al ${ }^{34}$ for the carbon dioxide. This singlesite model were previously evaluated for the calculation of thermodynamic and transport

properties of carbon dioxide in alkane mixtures. ${ }^{35136}$ All parameters of the employed force fields are available in Table S1 in the Supplementary Material.

Playmol ${ }^{37}$ was employed to construct cubic simulation boxes with $\mathrm{N}=1000$ molecules in total, and then LAMMPS ${ }^{\sqrt{38}}$ was used to carry out the MD simulations. These were 
performed by considering periodic boundary conditions and a $1 \mathrm{fs}$ timestep. The integration of the equations of motion was accomplished by means of the LAMMPS implementation of the algorithm originally described by Tuckerman et al. ${ }^{39}$ We used a Nosé-Hoover Chain with three thermostats ${ }^{40}$ with its characteristic time scale set to 100 fs to control the temperature, and the Martyna-Tobias-Klein barostat ${ }^{41}$ with characteristic time scale of 1000 fs to control the pressure.

The MD protocol started with a 7 ns simulation in the isothermal-isobaric ensemble (NPT) to stabilize the volume of the simulation box and determine the density of each system using the last 5 ns of this range. Then, new simulation boxes were assembled with Playmol employing the calculated densities. Finally, the systems were simulated for $1 \mathrm{~ns}$ in the canonical ensemble (NVT) for equilibration, followed by $5 \mathrm{~ns}$ in the same condition for data production. In the production step, the center of mass position of each molecule was stored at every $200 \mathrm{fs}$.

\section{Fick diffusion coefficients}

We calculated the MS coefficients through the well-established Onsager coefficient approach, using Eqs. (5) and (6). For attaining quality statistics, results were treated by the use of multiple origins of calculation. ${ }^{42}$ The MS coefficients were then converted to Fick coefficients through Eq. (3). We performed the calculation of the thermodynamic factor by the static structure factor approach in the Fourier domain, as presented in Eqs. (11) and (12). The thermodynamic limit condition $(q \rightarrow 0)$ was then obtained by extrapolating the static structure factor results through an analytical expression based on the Ornstein-Zernike direct correlation function, that is,, 3243

$$
S_{i j}^{-1}(q)=c_{0, i j}+c_{2, i j} q^{2}+c_{4, i j} q^{4}+\mathcal{O}\left(q^{6}\right),
$$


in which the odd-degree terms of the expansion are null and, consequently, the derivative of the series at $\mathrm{q}=0$ is zero. This is in line with the theoretical statement that static structure factors are even functions. As the simulated systems are isotropic, we averaged the $S_{i j}(\boldsymbol{q})$ values over all lattice vectors of equal magnitudes, which made the static structure factor dependent only on the module of lattice vectors, that is, $S_{i j}(q)$. We set a maximum assessment at $q_{c}=2 \pi L^{-1} \sqrt{20}$, which, after disregarding $q=0$, yields 388 unique pairs of lattice vectors $( \pm \boldsymbol{q})$. The choice of this cutoff is arbitrary, but a reasonable amount of vectors must be employed to allow extrapolation. Of course, the larger the cutoff, the higher even order terms will be needed in the expansion of the Ornstein-Zernike direct correlation function. For this extrapolation, the parameters $c_{0, i j}, c_{2, i j}, c_{4, i j}$ were adjusted using an optimization algorithm that employs the sum of quadratic residues as the objective function. $\cdot 44[46$

Regarding the determination of the Fick coefficients through the modified Fourier Correlation Method, we employed Eqs. (18), (25), and (26) to perform the calculations. As in the case of the static structure factor, the distinct-intermediate scattering function $\mathbb{S}_{D}(\boldsymbol{q}, t)$ was averaged over all equivalent lattice vectors, reducing the dependence of this property to the lattice vector module $q$. The Fick diffusion coefficient in the thermodynamic limit was obtained by extrapolating the results to $q \rightarrow 0$. By analogy, we assumed the same expansion of the Eq. 27) to perform extrapolation of the mutual diffusion coefficient, that is,

$$
D_{12}(q)=c_{0}^{*}+c_{2}^{*} q^{2}+c_{4}^{*} q^{4}+\mathcal{O}\left(q^{6}\right),
$$

where the coefficients of the correlation function may no longer assume physical values. Again, the extrapolation was based on 388 pairs of lattice vectors resulting from a cutoff $q_{c}=2 \pi L^{-1} \sqrt{20}$. The parameter adjustment was also performed through an optimization algorithm. 44.46

Finally, we also determined the Fick diffusion coefficients according to the original FCM, 
in order to compare the results with those obtained through the mFCM. In this case, we employed Eqs. (7), 10), and (13) to perform the original calculation as proposed by Nichols and Wheeler. ${ }^{22}$ The extrapolation procedure to determine diffusivities in the thermodynamic limit was the same as previously described, that is, using Eq. (28).

\section{Results and Discussion}

\section{Density and thermodynamic factor results}

The simulation density results presented a good agreement with the values obtained from the GERG-2008 equation of state, ${ }^{47}$ available in the REFPROP software package. ${ }^{48}$ The relative deviations between the MD results and those of REFPROP lie between $2 \%$ and $6 \%$. According to the estimates of critical coordinates also provided by REFPROP, all studied systems are above their critical pressures and below their critical temperatures. Therefore, they are in the compressible liquid phase region. The computed thermodynamic factors are always close to unit, which indicates that all evaluated mixtures present small deviations from ideality. The densities and thermodynamic factors obtained for all evaluated mixtures are listed in Tables S2-S4 in the Supplementary Material.

It is worth mentioning that the calculation of the thermodynamic factor through the Kirkwood-Buff formalism in the Fourier domain presented reasonable thermodynamic results, since the predicted behavior close to the thermodynamic ideality is expected in this case. Figure 1 illustrates the static structure factors obtained in the simulations, as well as the model fitting used to extrapolate the data, in the case of a $\mathrm{CO}_{2}+$ n-octane mixture with $x_{\mathrm{CO}_{2}}=0.6167$, at $290.0 \mathrm{~K}$ and $3.673 \mathrm{MPa}$. The extrapolation of the static structure factors via Eq. 27) seems quite satisfactory. Besides, the analytical extrapolation model based on the Ornstein-Zernike direct correlation function proved to describe the behavior of the structure factors accurately. Tables S5S57 in the Supplementary Material contain all the results for the static structure factors in the thermodynamic limit, $S_{i j}(0)$, used here to 
calculate thermodynamic factors.

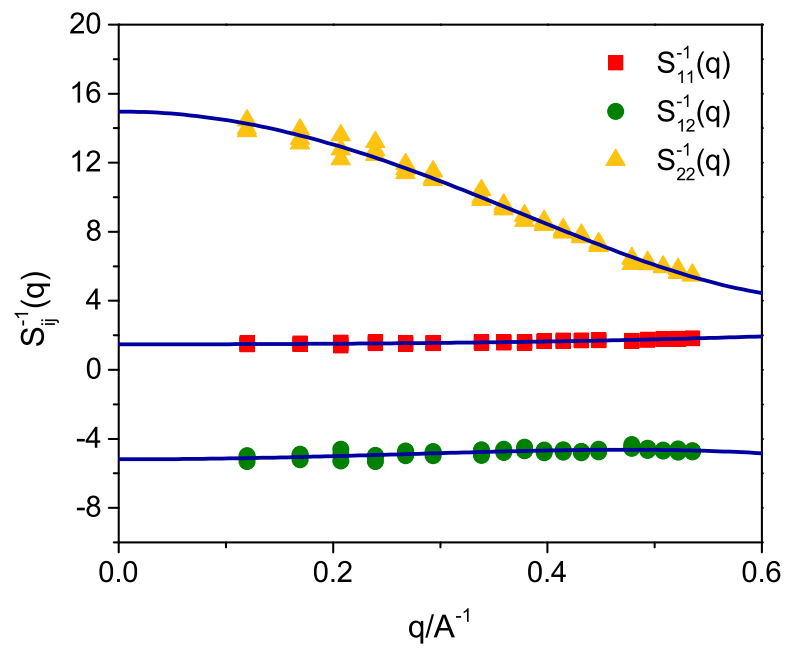

Figure 1: Static structure factors $S_{11}(q), S_{12}(q)$, and $S_{22}(q)$ in a $\mathrm{CO}_{2}+$ n-octane mixture, with $x_{\mathrm{CO}_{2}}=0.6167$, at $290.0 \mathrm{~K}$ and $3.673 \mathrm{MPa}$. - Extrapolation model according to Eq. (27).

It is also noteworthy that the choice of this methodology for the calculation of the thermodynamic factor is due to the fact that it arguably surmounts finite-size effects, commonly observed in the resolution of Kirkwood-Buff integrals in the canonical ensemble by means of radial distribution functions. $\frac{26-30 \mid 49}{2}$ Figure 2 illustrates the effect of the simulation box size on the determination of the static structure factor for a $\mathrm{CO}_{2}+$ n-octane mixture with $x_{\mathrm{CO}_{2}}=0.2427$ at $290.0 \mathrm{~K}$ and $1.265 \mathrm{MPa}$. Note that the static structure factors followed a well-defined curve, whose extrapolation to a value in the thermodynamic limit $(q \rightarrow 0)$ seems to be little dependent on the size of the simulated system. Of course, larger simulation boxes provide structure factor data closer to $q=0$, which makes the extrapolation more reliable. Similar results on the evaluation of the finite-size effect on the static structure factor have already been shown for a LJ mixture. ${ }^{30}$ On the other hand, it is clear that larger fluctuations occur close to $q=0$. This is so because, when $q \rightarrow 0$, there are fewer equivalent lattice vectors for determining the mean value of the structure factor. 

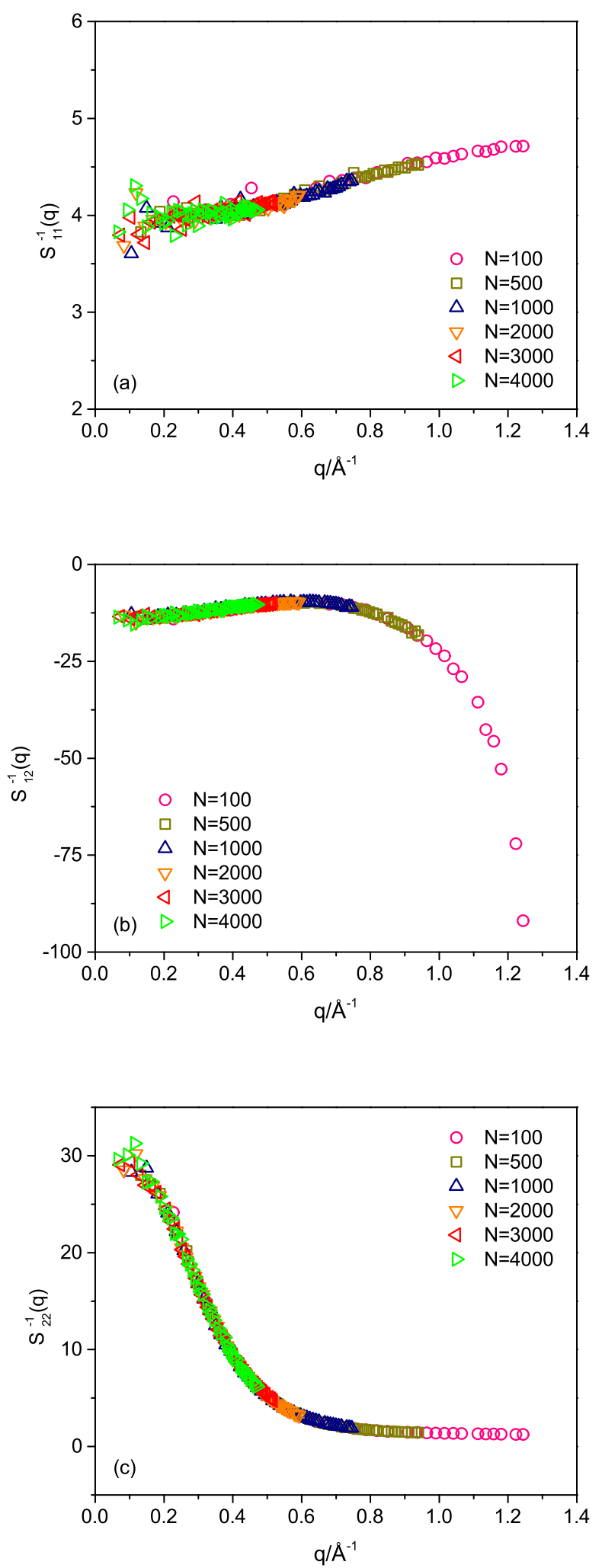

Figure 2: Static structure factors (a) $S_{11}(q)$, (b) $S_{12}(q)$, and (c) $S_{22}(q)$ at different simulation box sizes in a $\mathrm{CO}_{2}+$ n-octane mixture, with $x_{\mathrm{CO}_{2}}=0.2427$, at $290.0 \mathrm{~K}$ and $1.265 \mathrm{MPa}$. 


\section{Fick diffusion coefficients}

In this section, we discuss and compare the results of Fick diffusivities obtained by means of different equilibrium MD strategies, namely (i) the Onsager coefficient approach, associated to the thermodynamic factor $(\mathrm{OC}+\mathrm{TF})$, (ii) the new $\mathrm{mFCM}$, and (iii) the original FCM. All the results of mutual diffusion coefficients for the evaluated mixtures are listed in Tables 143 in the Supplementary Material. We compared our results to experimental data reported by Cadogan et al. ${ }^{50}$ for the mixtures at infinite dilution of $\mathrm{CO}_{2}$, and to those of Wang et al. $\quad(1996)^{51}$ for the mixtures of $\mathrm{CO}_{2}+\mathrm{n}$-octane at different concentrations. For illustrative purposes, a few examples of linear regression sets used for the calculation of the Onsager coefficients are also available in Figure S1 of the Supplementary Material. This methodology is well consolidated and will not be further discussed here.

In general, Fick coefficient results showed reasonable agreement to experimental data. However, as shown in Tables 173, the results obtained through the original FCM provided quite different Fick coefficients when considering each part of the self-intermediate scattering functions $\left(\phi_{11}\right.$ and $\left.\phi_{22}\right)$. Although employing the $\mathbb{S}_{11}(\boldsymbol{q}, t)$ part has provided physically meaningful results, it is essential to note that component 1 in the mixture $\left(\mathrm{CO}_{2}\right)$ was treated as a single Lennard-Jones particle. As the efficiency of the original FCM have already been demonstrated for LJ-like molecules, it was expected that the results provided by this term would be reasonable. On the other hand, the results obtained with the $\mathbb{S}_{22}(\boldsymbol{q}, t)$ part of the self-intermediate scattering function are not physically consistent. We attribute this issue to the fact that these other components of the mixtures are chain-like hydrocarbons (n-hexane or n-octane), whose long and dynamic structures may cause the self-intermediate scattering function related to the center of mass to rapidly increase in the thermodynamic limit. Thus, it is noted that the original FCM does not always provide satisfactory results for mixtures with these molecular compounds.

Figures 3 to 5 graphically show a comparison between the experimental data and the values obtained from our simulations through the $\mathrm{OC}+\mathrm{TF}$ and the $\mathrm{mFCM}$ approaches. 
Table 1: Fick diffusion coefficients for $\mathrm{CO}_{2}+$ n-hexane mixtures at infinity dilution of $\mathrm{CO}_{2}$, under different conditions of temperature and pressure. OC+TF: traditional approach of the Onsager coefficients; mFCM: modified Fourier Correlation Method; FCM: original Fourier Correlation Method.

\begin{tabular}{|c|c|c|c|c|c|c|c|c|c|c|}
\hline \multirow{3}{*}{$\mathbf{T} / \mathbf{K}$} & \multirow{3}{*}{$\mathrm{P} / \mathrm{MPa}$} & \multicolumn{9}{|c|}{$D_{12} / 10^{-9} m^{2} s^{-1}$} \\
\hline & & \multirow[b]{2}{*}{ Exp. } & \multirow[b]{2}{*}{$\mathrm{OC}+\mathrm{TF}$} & \multirow[b]{2}{*}{ Dev.(\%) } & \multirow[b]{2}{*}{ mFCM } & \multirow[b]{2}{*}{ Dev.(\%) } & \multicolumn{4}{|c|}{ FCM } \\
\hline & & & & & & & $\phi_{11}$ & Dev.(\%) & $\phi_{22}$ & Dev.(\%) \\
\hline \multirow{4}{*}{298.0} & 1.3 & $8.32 \pm 0.22$ & $7.22 \pm 1.31$ & $-13.20 \%$ & $7.63 \pm 0.01$ & $-8.35 \%$ & $7.29 \pm 0.01$ & $-12.39 \%$ & $61.45 \pm 0.01$ & $638.60 \%$ \\
\hline & 22.0 & $7.27 \pm 0.19$ & $6.19 \pm 0.41$ & $-14.86 \%$ & $5.47 \pm 0.01$ & $-24.82 \%$ & $6.31 \pm 0.01$ & $-13.22 \%$ & $49.05 \pm 0.01$ & $574.69 \%$ \\
\hline & 49.0 & $6.06 \pm 0.16$ & $5.18 \pm 0.33$ & $-14.54 \%$ & $5.23 \pm 0.01$ & $-13.74 \%$ & $5.60 \pm 0.01$ & $-7.56 \%$ & $37.31 \pm 0.01$ & $515.69 \%$ \\
\hline & 65.0 & $5.13 \pm 0.13$ & $4.93 \pm 0.66$ & $-3.81 \%$ & $5.16 \pm 0.01$ & $0.60 \%$ & $5.19 \pm 0.01$ & $1.10 \%$ & $36.02 \pm 0.01$ & $602.19 \%$ \\
\hline \multirow{4}{*}{323.0} & 1. & $10.50 \pm 0.27$ & $9.04 \pm 1.10$ & $-13.90 \%$ & $8.84 \pm 0.01$ & $-15.79 \%$ & $9.31 \pm 0.01$ & $-11.38 \%$ & $67.46 \pm 0.01$ & $542.50 \%$ \\
\hline & 22.0 & $9.27 \pm 0.24$ & $8.25 \pm 0.38$ & $-11.05 \%$ & $7.38 \pm 0.01$ & $-20.34 \%$ & $7.92 \pm 0.01$ & $-14.54 \%$ & $50.47 \pm 0.01$ & $444.47 \%$ \\
\hline & 50.0 & $7.61 \pm 0.20$ & $7.03 \pm 0.46$ & $-7.56 \%$ & $6.33 \pm 0.01$ & $-16.79 \%$ & $6.70 \pm 0.01$ & $-11.95 \%$ & $44.90 \pm 0.01$ & $490.02 \%$ \\
\hline & 66.0 & $7.15 \pm 0.19$ & $6.44 \pm 0.53$ & $-9.88 \%$ & $6.37 \pm 0.01$ & $-10.90 \%$ & $6.31 \pm 0.01$ & $-11.71 \%$ & $44.68 \pm 0.01$ & $524.90 \%$ \\
\hline \multirow{4}{*}{373.0} & 1. & $15.80 \pm 0.41$ & $13.58 \pm 1.48$ & $-14.05 \%$ & $12.07 \pm 0.01$ & $-23.61 \%$ & $14.01 \pm 0.01$ & $-11.30 \%$ & $53.04 \pm 0.01$ & $235.67 \%$ \\
\hline & 22.0 & $13.40 \pm 0.35$ & $12.23 \pm 0.83$ & $-8.76 \%$ & $11.35 \pm 0.01$ & $-15.32 \%$ & $12.12 \pm 0.01$ & $-9.53 \%$ & $51.54 \pm 0.01$ & $284.62 \%$ \\
\hline & 49.0 & $11.00 \pm 0.29$ & $9.43 \pm 1.45$ & $-14.32 \%$ & $9.60 \pm 0.01$ & $-12.74 \%$ & $10.08 \pm 0.01$ & $-8.37 \%$ & $54.30 \pm 0.01$ & $393.65 \%$ \\
\hline & 64.0 & $10.30 \pm 0.27$ & $8.94 \pm 0.92$ & $-13.25 \%$ & $8.54 \pm 0.01$ & $-17.06 \%$ & $8.91 \pm 0.01$ & $-13.50 \%$ & $44.97 \pm 0.01$ & $336.56 \%$ \\
\hline
\end{tabular}

Table 2: Fick diffusion coefficients for $\mathrm{CO}_{2}+$ n-octane mixtures at infinity dilution of $\mathrm{CO}_{2}$, under different conditions of temperature and pressure. $\mathrm{OC}+\mathrm{TF}$ : traditional approach of the Onsager coefficients; mFCM: modified Fourier Correlation Method; FCM: original Fourier Correlation Method.

\begin{tabular}{cc|c|cc|cc|cccc}
\hline \multirow{2}{*}{ T/K } & P/MPa & \multicolumn{10}{|c}{$D_{12} / 10^{-9} m^{2} s^{-1}$} \\
\cline { 3 - 11 } & & Exp. & OC+TF & Dev.(\%) & mFCM & Dev.(\%) & $\phi_{11}$ & Dev.(\%) & $\phi_{22}$ & Dev.(\%) \\
\hline & 1.0 & $6.32 \pm 0.16$ & $5.12 \pm 0.25$ & $-18.91 \%$ & $4.50 \pm 0.01$ & $-28.75 \%$ & $4.85 \pm 0.01$ & $-23.26 \%$ & $56.93 \pm 0.01$ & $800.76 \%$ \\
298.0 & 10.0 & $5.66 \pm 0.15$ & $4.59 \pm 0.77$ & $-18.93 \%$ & $4.02 \pm 0.01$ & $-28.90 \%$ & $4.49 \pm 0.01$ & $-20.64 \%$ & $47.59 \pm 0.01$ & $740.84 \%$ \\
& 31.0 & $4.72 \pm 0.12$ & $3.93 \pm 0.46$ & $-16.70 \%$ & $3.78 \pm 0.01$ & $-19.84 \%$ & $4.18 \pm 0.01$ & $-11.42 \%$ & $42.08 \pm 0.01$ & $791.54 \%$ \\
& 50.0 & $4.28 \pm 0.11$ & $3.18 \pm 0.41$ & $-25.74 \%$ & $3.34 \pm 0.01$ & $-21.99 \%$ & $3.66 \pm 0.01$ & $-14.59 \%$ & $39.69 \pm 0.01$ & $827.25 \%$ \\
& 68.0 & $3.83 \pm 0.10$ & $3.28 \pm 0.15$ & $-14.25 \%$ & $3.36 \pm 0.01$ & $-12.27 \%$ & $3.36 \pm 0.01$ & $-12.30 \%$ & $30.32 \pm 0.01$ & $691.75 \%$ \\
\hline & 1.0 & $8.19 \pm 0.21$ & $7.19 \pm 0.45$ & $-12.18 \%$ & $6.82 \pm 0.01$ & $-16.72 \%$ & $6.76 \pm 0.01$ & $-17.52 \%$ & $58.76 \pm 0.01$ & $617.44 \%$ \\
323.0 & 10.0 & $7.36 \pm 0.19$ & $6.20 \pm 0.44$ & $-15.71 \%$ & $5.83 \pm 0.01$ & $-20.84 \%$ & $6.01 \pm 0.01$ & $-18.33 \%$ & $55.98 \pm 0.01$ & $660.63 \%$ \\
& 30.0 & $6.10 \pm 0.16$ & $4.99 \pm 0.40$ & $-18.14 \%$ & $4.75 \pm 0.01$ & $-22.15 \%$ & $5.10 \pm 0.01$ & $-16.42 \%$ & $48.54 \pm 0.01$ & $695.69 \%$ \\
& 51.0 & $5.62 \pm 0.15$ & $4.42 \pm 0.38$ & $-21.41 \%$ & $4.42 \pm 0.01$ & $-21.36 \%$ & $4.36 \pm 0.01$ & $-22.46 \%$ & $40.09 \pm 0.01$ & $613.31 \%$ \\
& 68.0 & $5.10 \pm 0.13$ & $4.18 \pm 0.34$ & $-18.08 \%$ & $4.14 \pm 0.01$ & $-18.87 \%$ & $4.24 \pm 0.01$ & $-16.94 \%$ & $43.33 \pm 0.01$ & $749.69 \%$ \\
\hline
\end{tabular}

Table 3: Fick diffusion coefficients for $\mathrm{CO}_{2}+$ n-octane mixtures at different compositions, temperatures, and pressures. $\mathrm{OC}+\mathrm{TF}$ : traditional approach of the Onsager coefficients; mFCM: modified Fourier Correlation Method; FCM: original Fourier Correlation Method.

\begin{tabular}{|c|c|c|c|c|c|c|c|c|c|c|c|}
\hline \multirow{3}{*}{$\mathbf{T} / \mathbf{K}$} & \multirow{3}{*}{$x_{\mathrm{CO}_{2}}$} & \multirow{3}{*}{$\mathbf{P} / \mathbf{M P a}$} & \multicolumn{9}{|c|}{$D_{12} / 10^{-9} m^{2} s^{-1}$} \\
\hline & & & \multirow[b]{2}{*}{ Exp. } & \multirow[b]{2}{*}{$\mathrm{OC}+\mathrm{TF}$} & \multirow[b]{2}{*}{ Dev.(\%) } & \multirow[b]{2}{*}{ mFCM } & \multirow{2}{*}{ Dev.(\%) } & \multicolumn{4}{|c|}{ FCM } \\
\hline & & & & & & & & $\phi_{11}$ & Dev.(\%) & $\phi_{22}$ & Dev.(\%) \\
\hline \multirow{5}{*}{290.0} & 0.2427 & 1.265 & $4.58 \pm 0.46$ & $4.33 \pm 0.20$ & $-5.48 \%$ & $4.47 \pm 0.01$ & $-2.40 \%$ & $4.73 \pm 0.01$ & $3.24 \%$ & $7.81 \pm 0.01$ & $70.63 \%$ \\
\hline & 0.3324 & 1.843 & $2.79 \pm 0.28$ & $4.88 \pm 0.47$ & $74.89 \%$ & $5.11 \pm 0.01$ & $83.24 \%$ & $5.33 \pm 0.01$ & $91.26 \%$ & $7.77 \pm 0.01$ & $178.75 \%$ \\
\hline & 0.4124 & 2.358 & $3.42 \pm 0.34$ & $4.90 \pm 0.25$ & $43.35 \%$ & $4.80 \pm 0.01$ & $40.40 \%$ & $5.05 \pm 0.01$ & $47.80 \%$ & $6.35 \pm 0.01$ & $85.61 \%$ \\
\hline & 0.5216 & 3.061 & $3.99 \pm 0.40$ & $4.70 \pm 0.25$ & $17.79 \%$ & $4.93 \pm 0.01$ & $23.60 \%$ & $5.30 \pm 0.01$ & $32.81 \%$ & $5.91 \pm 0.01$ & $48.13 \%$ \\
\hline & 0.6167 & 3.673 & $5.31 \pm 0.53$ & $5.27 \pm 0.59$ & $-0.84 \%$ & $5.23 \pm 0.01$ & $-1.53 \%$ & $5.58 \pm 0.01$ & $4.99 \%$ & $5.99 \pm 0.01$ & $12.71 \%$ \\
\hline \multirow{5}{*}{311.0} & 0.1344 & 0.747 & $4.96 \pm 0.50$ & $6.05 \pm 0.37$ & $22.06 \%$ & $5.57 \pm 0.01$ & $12.32 \%$ & $5.91 \pm 0.01$ & $19.34 \%$ & $14.43 \pm 0.01$ & $191.21 \%$ \\
\hline & 0.2384 & 1.667 & $6.92 \pm 0.69$ & $5.75 \pm 0.77$ & $-16.82 \%$ & $5.77 \pm 0.01$ & $-16.53 \%$ & $5.99 \pm 0.01$ & $-13.42 \%$ & $9.54 \pm 0.01$ & $37.95 \%$ \\
\hline & 0.2499 & 1.769 & $8.11 \pm 0.81$ & $6.04 \pm 0.37$ & $-25.49 \%$ & $5.80 \pm 0.01$ & $-28.46 \%$ & $6.00 \pm 0.01$ & $-25.94 \%$ & $9.67 \pm 0.01$ & $19.34 \%$ \\
\hline & 0.2814 & 2.048 & $3.98 \pm 0.40$ & $6.21 \pm 0.64$ & $55.95 \%$ & $5.75 \pm 0.01$ & $44.36 \%$ & $6.03 \pm 0.01$ & $51.36 \%$ & $8.88 \pm 0.01$ & $122.94 \%$ \\
\hline & 0.4007 & 3.103 & $4.61 \pm 0.46$ & $6.25 \pm 0.15$ & $35.73 \%$ & $5.95 \pm 0.01$ & $29.27 \%$ & $6.31 \pm 0.01$ & $36.94 \%$ & $7.82 \pm 0.01$ & $69.69 \%$ \\
\hline
\end{tabular}


Figures 3 and 4 show that, in the case of $\mathrm{CO}_{2}+$ n-hexane and $\mathrm{CO}_{2}+$ n-octane mixtures at infinity dilution of $\mathrm{CO}_{2}$, both the Onsager coefficient approach and the mFCM showed good agreement with the experimental points. The values calculated by the traditional method were sometimes higher and sometimes lower than those calculated by the mFCM, but both approaches provided underestimated results when compared to the experimental data. This finding can be explained by the residual finite-size effect of the simulation box, which does not allow a proper account for the long-range hydrodynamic interactions and, mainly, because of the natural limitation of the force field employed, whose parameterization was not targeted to reproduce mutual diffusivity.

Figure 5 shows that, in the case of the $\mathrm{CO}_{2}+$ n-octane mixtures at different temperatures, pressures, and concentrations of $\mathrm{CO}_{2}$, the obtained results do not present a good agreement to experimental points. However, these experimental data are quite oscillatory and their uncertainties are not known. We considered a scenario in which the error bars are $10 \%$ of the property value, as the author of the original paper ${ }^{51}$ suggests. In these cases, results obtained through the MD methodologies seem to have agreed with the experimental data only at the extremes of the evaluated concentrations, with no agreement in the middle points. It is important to highlight, however, that both the $\mathrm{mFCM}$ and the traditional methodology of Onsager coefficients presented consonant results, with a smooth and physically consistent profile.

To illustrate the process of determining the Fick diffusion coefficient through the mFCM, Figure 6 shows $\phi_{D}$ at different wave numbers, together with the linear regression proposed in Equation (26) to determine the mutual diffusion coefficient in each case. These curves decay because the real parts of both the self- and distinct-intermediate scattering functions tend to zero, as this quantity describes the microscopic thermal fluctuations of the system which should, according to the hypothesis of Onsager regression, decay to zero over time just like macroscopic perturbations in a non-equilibrium condition. ${ }^{22152]}$ Figure 6(a) shows the behavior of $\phi_{D}$ at a low $q$-value, where a nonlinear behavior is noted for short time lags, 

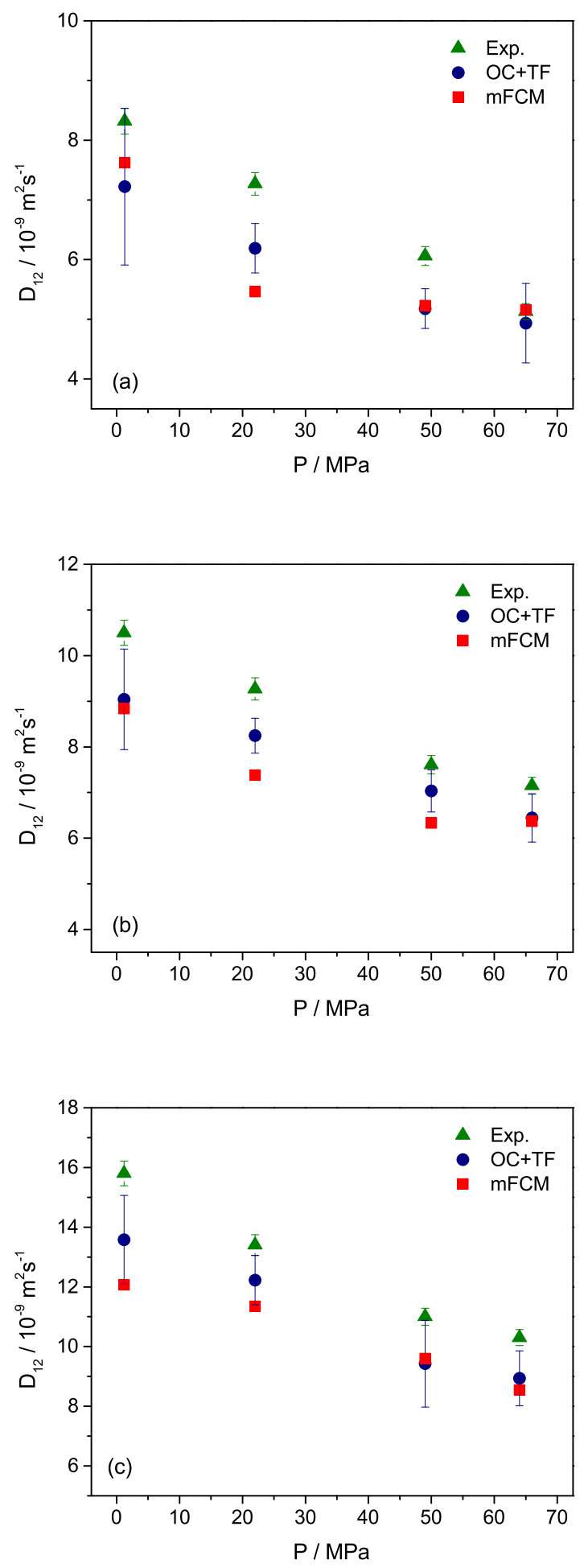

Figure 3: Fick diffusion coefficients for $\mathrm{CO}_{2}+$ n-hexane mixtures at infinity dilution of $\mathrm{CO}_{2}$, at (a) $298.0 \mathrm{~K}$, (b) $323.0 \mathrm{~K}$, and (c) $373.0 \mathrm{~K}$. $\triangle$ Experimental data from Cadogan et al. (2016); $\bigcirc$ Molecular Dynamics results through Onsager coefficients with thermodynamic factors; $\square$ Molecular Dynamics results through the modified Fourier Correlation Method. 

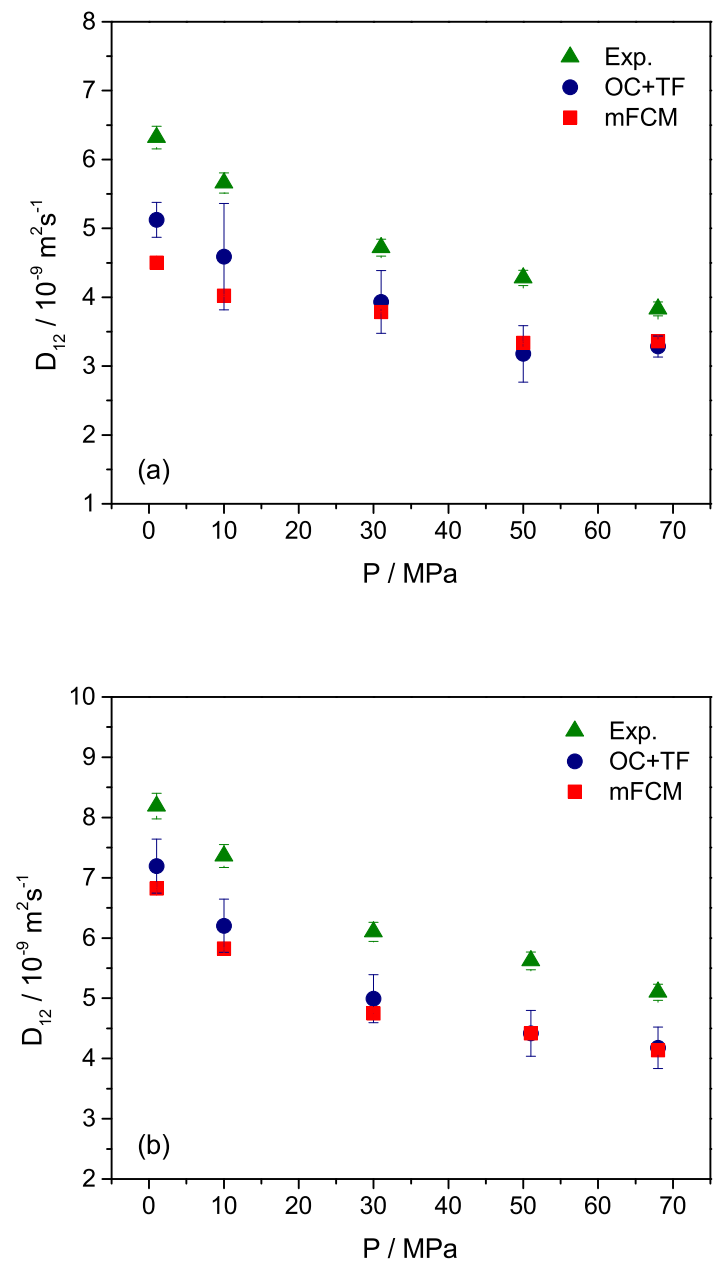

Figure 4: Fick diffusion coefficients for $\mathrm{CO}_{2}+$ n-octane mixtures at infinity dilution of $\mathrm{CO}_{2}$, at (a) $298.0 \mathrm{~K}$, and (b) $323.0 \mathrm{~K}$. $\triangle$ Experimental data from Cadogan et al. (2016); $\bigcirc$ Molecular Dynamics results through Onsager coefficients with thermodynamic factors; $\square$ Molecular Dynamics results through the modified Fourier Correlation Method. 

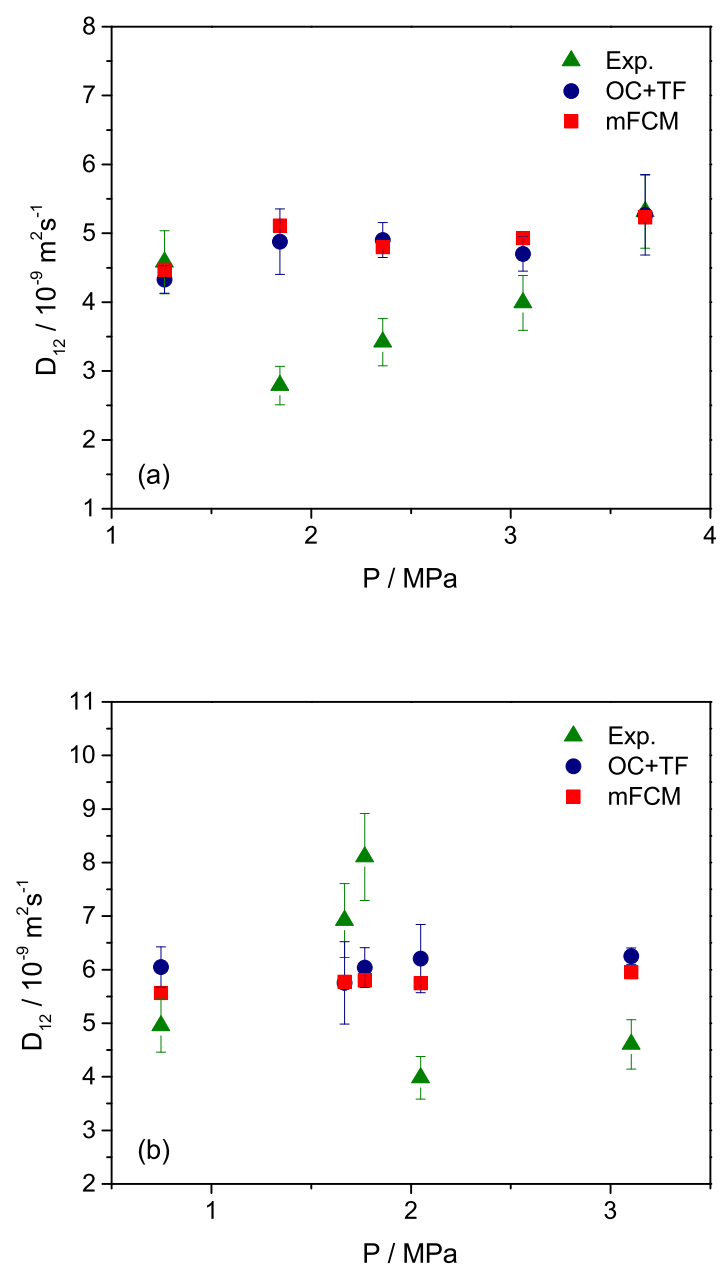

Figure 5: Fick diffusion coefficients for $\mathrm{CO}_{2}+$ n-octane mixtures at different pressures and concentrations, at (a) $290.0 \mathrm{~K}$, and (b) $311.0 \mathrm{~K}$. $\triangle$ Experimental data from Wang et al., with error bars estimated by $10 \%$ of the property value; $\bigcirc$ Molecular Dynamics results through Onsager coefficients with thermodynamic factors; $\square$ Molecular Dynamics results through the modified Fourier Correlation Method. 
which rapidly decays and converges to a linear trend for longer time lags. We believe that this nonlinearity at low time lags is a result of the ballistic effects, when molecular collisions still play a role in diffusion. This effect is also commonly observed in measurements not related to the Fourier domain, as in the case of the exponential behavior observed at the beginning of the Mean Square Displacement curve (MSD). For the purpose of linear regression, the initial part of the curve with inertial effects was disregarded. Nichols and Wheeler ${ }^{22}$ predicted that this behavior should be observed for low $q$-values in the dynamic structure factor, although they have not effectively observed the phenomenon in the case of simple LennardJones fluids. Figure 6(b) shows the behavior of $\phi_{D}$ for an intermediate $q$-value, where the ballistic effects at the beginning of the curve are little pronounced and the trend of the data is practically linear, as expected. Finally, Figure 6(c) illustrates the behavior of $\phi_{D}$ for a high $q$-value, where a nonlinear decay is observed for long periods of time. In this case, the slower decay is observed after a certain characteristic time in which the hydrodynamic interactions promote a relaxation in the system, which causes a logarithmic decay of the intermediate scattering function. $\frac{52]}{}$ Again, only the linear region was taken into account in the fitting for the computation of the mutual diffusion coefficient corresponding to this wave number. In this work, we implemented an automatic routine for the linearization of $\phi_{D}$ at different $q$-values. This routine considers the peculiarities observed for different magnitudes of the lattice vector and performs the regression in the region of intermediate times within the studied time window, which ensures that the linear fit always show a quadratic Pearson correlation coefficient greater than $0.95\left(R^{2}=0.95\right)$. Another possible strategy would be to estimate the characteristic time for each case and limit the linear regression to that time window, being only necessary to discard the regions where the initial ballistic effects are relevant.

It is important to emphasize the difficulty in determining the time-lag of the exponential decay of $\phi_{D}(q, t)$ data for different $q$-values. In the limit of $q \rightarrow \infty$, it is expected that the non-linear decay of $\phi_{D}$ starts before and extends for longer intervals, which represents an 



Figure 6: Linear regression of $\phi_{D}$ at (a) $q=0.1197 \AA^{-1}$, (b) $q=0.3385 \AA^{-1}$, and (c) $q=0.6533 \AA^{-1}$ in a $\mathrm{CO}_{2}+$ n-octane mixture, with $x_{\mathrm{CO}_{2}}=0.6167$, at $290.0 \mathrm{~K}$ and $3.673 \mathrm{MPa}$. $\square$ Molecular Dynamics results; - Linear regression according to Equation (26). 
intrinsic problem for the linear data regression. However, it must be considered that the results at high values of $q$ are not of great importance, since the only values that matters for the method are in the opposite extreme, that is, in the extrapolation for $q \rightarrow 0$. Also, in the limit of $q \rightarrow \infty$ the hypothesis of macroscopic diffusion loses validity and the results become less reliable.

Figure 7 exemplifies the extrapolation of the Fick diffusion coefficient obtained by the mFCM as a function of the wave numbers. The assumption that the Ornstein-Zernike correlation function would also be appropriate for the extrapolation of the mutual diffusivity seems to be valid, since the fitting to the simulation data was quite satisfactory in the context of the mFCM. On the other hand, Figure 8 shows the extrapolation of Fick coefficients obtained through the original FCM, employing both terms $\mathbb{S}_{11}(\boldsymbol{q}, t)$ and $\mathbb{S}_{22}(\boldsymbol{q}, t)$ of the selfintermediate scattering function. Again, it is noted that these two terms provided distinct results, which impairs the applicability of the original FCM for mixtures with molecular components as compared to Lennard-Jones fluids. Moreover, we observed that this difference gets bigger as the system gets richer in the n-alkane, that is, the problem is aggravated in limit of infinite dilution of $\mathrm{CO}_{2}$.

It is important to point out that the dispersion of the diffusivity values as a function of $q$ can be attenuated by statistical improvement, i.e. longer simulations. The uncertainties of the Fick coefficients determined from both FCM and mFCM were estimated based on the confidence interval of the adjusted parameters in the regression model, which ultimately turns this error into an indirect measurement of the degree of dispersion of the $D_{12}(q)$ data. Despite the clear dispersion on the $q$-dependent data, the estimated uncertainty on the Fick coefficient extrapolated to the thermodynamic limit is low.

A pertinent source of criticism, as highlighted by Nichols and Wheeler, $\stackrel{22}{2}$ is that both the FCM and the mFCM require extrapolation of the simulation data, which demands an appropriate model that must be able to describe the Fick diffusivities as a function of the $q$ values. On the other hand, one should keep in mind that other equilibrium-based approaches 


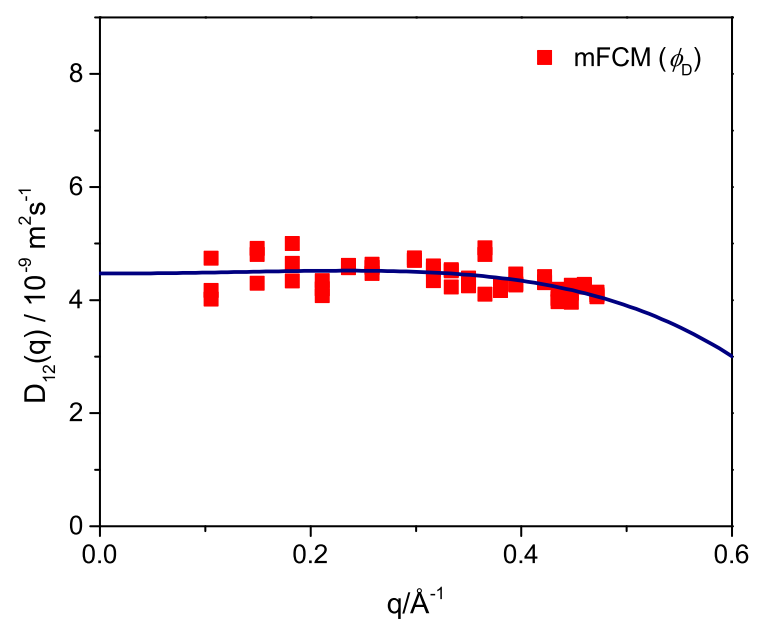

Figure 7: Fick diffusion coefficients as a function of the wave number in a $\mathrm{CO}_{2}+$ n-octane mixture, with $x_{\mathrm{CO}_{2}}=0.6167$, at $290.0 \mathrm{~K}$ and $3.673 \mathrm{MPa}$. $\square$ Molecular Dynamics results through the modified Fourier Correlation Method; - Extrapolation model according to Equation (28).

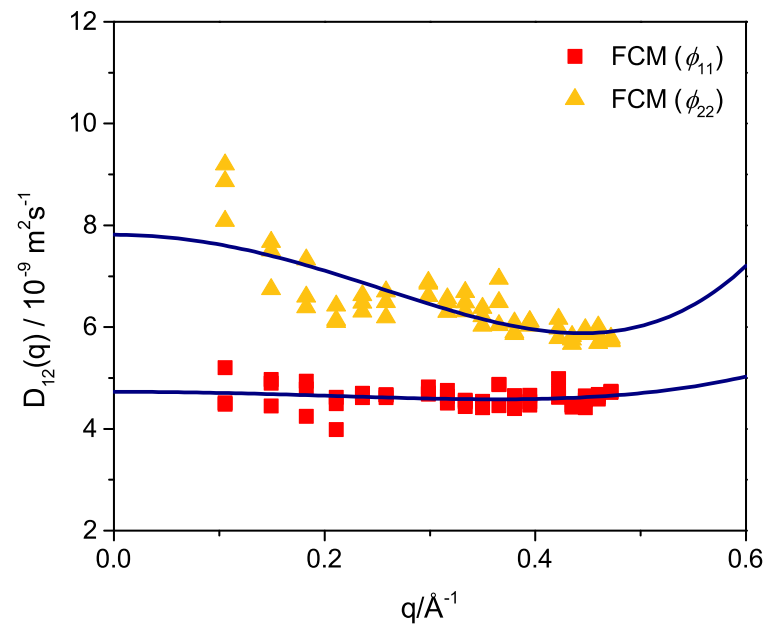

Figure 8: Fick diffusion coefficients as a function of the wave number in a $\mathrm{CO}_{2}+$ n-octane mixture, with $x_{\mathrm{CO}_{2}}=0.6167$, at $290.0 \mathrm{~K}$ and 3.673 MPa. $\square$ Molecular Dynamics results through the original Fourier Correlation Method; - Extrapolation model according to Equation (28). 
also need to assume an extrapolation to the thermodynamic limit, as in the case of the Onsager methodology (Eq. (5)). Besides, these well-established approaches do not directly provide the value of Fick coefficients, thus requiring the calculation of the thermodynamic factor, which is usually not trivial. In this sense, the mFCM approach not only directly provides the value of the Fick coefficient but also assumes an extrapolation to $q \rightarrow 0$, which provides Fick diffusivities less prone to the finite-size effect of the simulation boxes with periodic boundary conditions. This effect is discussed in the following section.

\section{Finite-size effect on Fick diffusion coefficients}

In this section, we evaluated the finite-size effect on the approaches we employed to estimate Fick diffusion coefficients. It is known that the Maxwell-Stefan coefficients determined by the traditional approach from Onsager coefficients may be subject to finite-size effects. .53 The thermodynamic factor that converted the Maxwell-Stefan coefficients to Fick diffusivities, however, is less prone to the finite-size effect of the simulation box as previously discussed. On the other hand, the Fick diffusivity determined through the mFCM theoretically presents no finite-size effect because the extrapolation to $q=0$ is supposed to provide mutual diffusion coefficients at the thermodynamic limit.

Figure 9 illustrates the effect of the simulation box size on the determination of the Fick coefficient through the $\mathrm{mFCM}$ for a $\mathrm{CO}_{2}+$ n-octane mixture with $x_{\mathrm{CO}_{2}}=0.2427$ at $290.0 \mathrm{~K}$ and 1.265 MPa. Note that the tendency of the Fick diffusivity value at the thermodynamic limit is the same for different system sizes. However, the dispersion of the data at different simulation box sizes cannot be neglected, as these oscillations may cause slightly different Fick coefficient values after the extrapolation process. It is worth mentioning that all data obtained from different simulation boxes collapse to a single curve, thus confirming the principle that $D_{12}$ actually depends on the wave number $q$ regardless of the system size. Evidently, simulating larger systems enable us to obtain estimates at smaller $q$ values and, as a consequence, the extrapolation to $q \rightarrow 0$ becomes more reliable. 


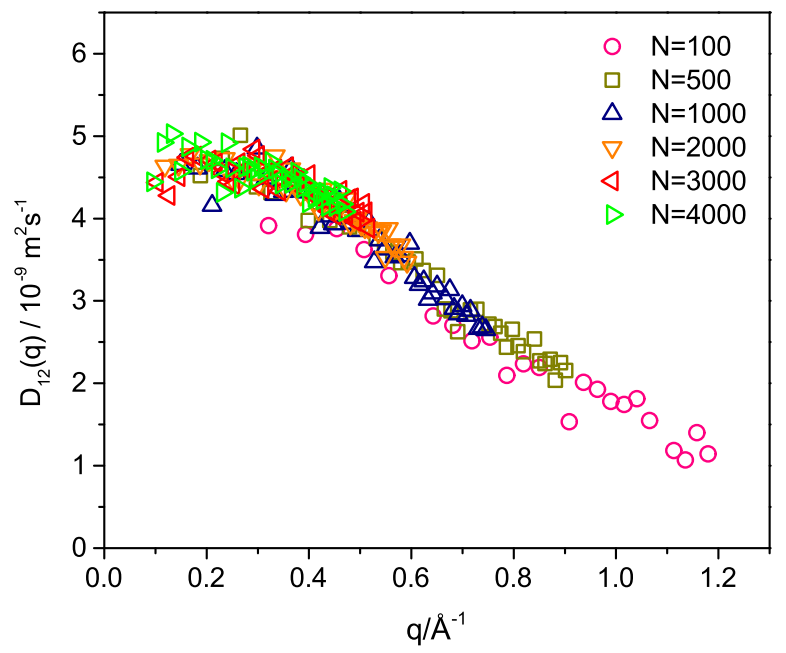

Figure 9: Fick diffusion coefficients as a function of the wave number at different simulation box sizes in a $\mathrm{CO}_{2}+\mathrm{n}$-octane mixture, with $x_{\mathrm{CO}_{2}}=0.2427$, at $290.0 \mathrm{~K}$ and $1.265 \mathrm{MPa}$. These results were obtained with the $\mathrm{mFCM}$.

In Figure 10, we directly compare the Fick coefficients obtained by the two approaches studied in this work, and we also include the Fick coefficients determined by the traditional methodology and corrected for finite-size effects according to a recent proposal made by Jamali et al. ${ }^{53}$ Such strategy has already been validated in the original work as well as in another recent study. ${ }^{[54}$ It basically consists in the use of the Yeh-Hummer factor $\stackrel{55}{\sqrt{55}}$ to correct the finite-size effects on the Fick diffusion coefficients, according to the equation

$$
D_{i j, \infty}=D_{i j}+\frac{\zeta k_{B} T}{6 \pi \eta L}
$$

where $D_{i j, \infty}$ is the Fick coefficient in the thermodynamic limit, $D_{i j}$ is the value obtained via EMD simulations by methods liable to finite-size effects, $\eta$ is the kinematic viscosity, and $\zeta \approx 2.837297 .[55156]$ We calculated the kinematic viscosity through the well-known Green-Kubo formalism by using a $7 \mathrm{~ns}$ production in the isothermal-isobaric ensemble (NpT) with 4000 molecules in the system. We also used a multiple-origin algorithm to statistically improve the obtained autocorrelation functions. The final result was obtained as an average of the 
results from triplicate simulations. For this $\mathrm{CO}_{2}+$ n-octane mixture with $x_{\mathrm{CO}_{2}}=0.2427$, at 290.0 $\mathrm{K}$ and 1.265 $\mathrm{MPa}$, the determined viscosity was $\eta=(3.48 \pm 0.12) 10^{-4} \mathrm{~Pa} \cdot \mathrm{s}$.

The diffusivities obtained by the traditional methodology through Onsager coefficients seem to be influenced by the size of the simulation box, which may indicate that long-range hydrodynamic interactions not properly accounted in periodic and finite systems tends to underestimate the calculated mutual diffusivity. However, it should be considered that in some cases the underestimation observed in the Fick coefficient by the traditional approach appears to be in the same order of magnitude as the uncertainties of the measurements itself. On the other hand, the new mFCM seems to considerably reduce the finite-size effect on the calculation of the Fick coefficients. As expected, the fluctuations in $D_{12}(q)$ observed in Figure 9 for different system sizes were sufficient to provide slightly different Fick coefficients for each case. Because of these fluctuations at different simulation box sizes, the mFCM as used in the present work does not seem to provide results completely free of the finite-size effects. However, we believe that longer simulations and greater accuracy in determining the linear region of the dynamic structure factor should reduce these fluctuations and make Fick diffusivities even less dependent on the simulation box size through the mFCM.

In general, the Jamali et al. ${ }^{53}$ correction greatly attenuates the effect of finite size of the simulation box, although the problem does not seem to be completely solved since the slope of its fitted line is not exactly zero. Thus, it is possible to state that the capacity of the mFCM to mitigate the finite-size effect on the calculation of the Fick coefficients is comparable to the one provided by the correction factor proposed by Jamali et al. $\frac{53}{5}$ The regression lines of the coefficients obtained by both approaches are practically parallel, and although the final values at the thermodynamic limit are slightly different from one approach to the other, the values are statistically equivalents if we consider the error bars. 


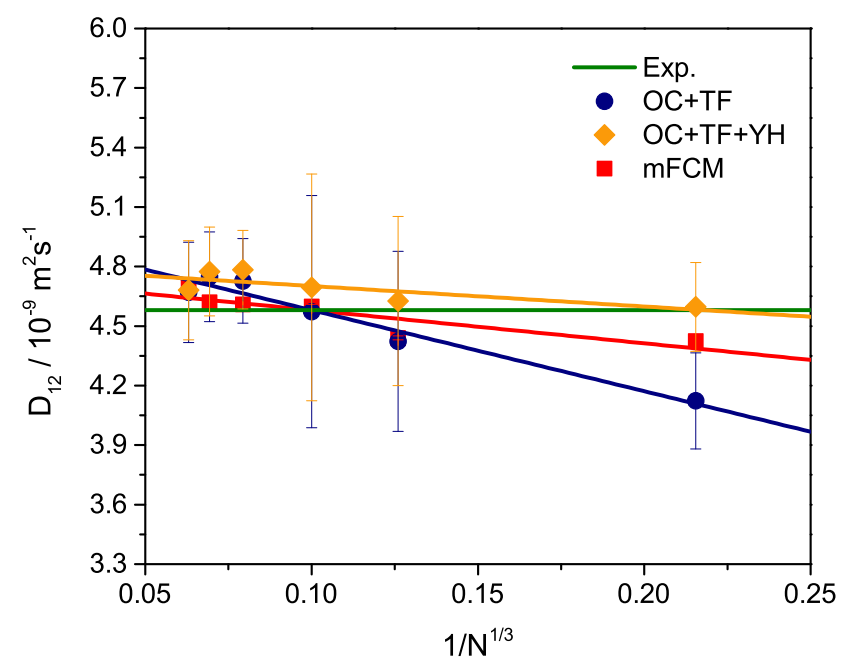

Figure 10: Fick diffusion coefficients as a function of the cubic simulation box size, for a $\mathrm{CO}_{2}+$ n-octane mixture, with $x_{\mathrm{CO}_{2}}=0.2427$, at $290.0 \mathrm{~K}$ and $1.265 \mathrm{MPa}$. $\bigcirc \mathrm{OC}+\mathrm{TF}$ : results through Onsager coefficients; $\diamond \mathrm{OC}+\mathrm{TF}+\mathrm{YH}$ : results through Onsager coefficients and corrected by the Yeh-Hummer factor; $\square$ mFCM: results through the modified Fourier Correlation Method; - Experimental data from Wang et al. (1996).

\section{Conclusions}

We presented an alternative approach to directly calculate Fick diffusion coefficients from equilibrium MD simulations, named the modified Fourier Correlation Method (mFCM). The new approach is a generalization of the original Fourier Correlation Method introduced by Nichols and Wheeler (2015),, 22 which succeeds in the case of simple Lennard-Jones fluids, but fails to provide suitable results in the case of binary molecular mixtures. To validate our approach, we performed the calculation of Fick diffusion coefficients for several $\mathrm{CO}_{2}+$ n-hexane and $\mathrm{CO}_{2}+$ n-octane mixtures at high pressures and at different temperatures. We obtained results with good agreement to experimental data and to Fick coefficients calculated through the traditional approach based on Onsager coefficients associated with the thermodynamic factor. Moreover, our new proposal proved to be a simpler alternative to the traditional methodology, since Fick diffusivities are obtained directly from a single calculation.

We also evaluated the effect of the simulation box size on the Fick coefficient obtained via 
molecular simulations. The well-established methodology that employs the Maxwell-Stefan (MS) coefficients converted to Fick diffusivities by the thermodynamic factor seemed to be subject to finite-size effects. In contrast, the new mFCM presented practically no finite-size issues, which is mainly caused by the dispersion of the $D_{12}(q)$ values that ultimately influences the data regression process. In general, the small effect observed in the results obtained by the $\mathrm{mFCM}$ is comparable to that observed in the Fick coefficients after application of a correction factor as proposed by Jamali et al. (2018).

In summary, the mFCM proved to be a reliable approach for calculating Fick diffusion coefficients in binary systems. Among its main advantages, we highlight the implicit incorporation of the thermodynamic factor in the calculation and the already mentioned mitigation of finite-size effects. On the other hand, issues concerning the necessity of extrapolation represent an inherent difficulty of the method.

Finally, it is worth mentioning that we were able to successfully calculate the thermodynamic factor through the static structure factor in the Fourier Domain. The proposed analytical model based on the Ornstein-Zernike formalism proved to be quite effective for extrapolating the results to the thermodynamic limit. In addition, this approach has been shown to be virtually immune to the finite-size effect of the simulation box, which is a problem commonly observed in Kirkwood-Buff integrals resolution.

\section{Acknowledgements}

The authors are grateful for the financial support provided by CAPES (finance code 001), Petrobras (project code CENPES 20934-6), and CNPq. We are also grateful for the help provided by Dr. Iuri Segtovich and for the computational resources provided by the Lobo-Carneiro Supercomputer Faculty at the Federal University of Rio de Janeiro, Brazil. 


\section{References}

(1) Vella, J. R. Fick Diffusion Coefficients of the Gaseous $\mathrm{CH}_{4}-\mathrm{CO}_{2}$ System from Molecular Dynamics Simulations Using TraPPE Force Fields at 101.325, 506.625, 1013.25, 2533.12, and 5066.25 kPa. Journal of Chemical \& Engineering Data 2019,

(2) Krishna, R.; van Baten, J. M. Describing diffusion in fluid mixtures at elevated pressures by combining the Maxwell-Stefan formulation with an equation of state. Chemical Engineering Science 2016, 153, 174-187.

(3) Hänggi, P.; Marchesoni, F. Introduction: 100 years of Brownian motion. Chaos 2005, 15.

(4) Cecconi, F.; Cencini, M.; Falcioni, M.; Vulpiani, A. Brownian motion and diffusion: From stochastic processes to chaos and beyond. Chaos 2005, 15.

(5) de Groot, S. R.; Mazur, P. Non-Equilibrium Thermodynamics; Dover Publications, Inc.: New York, 1984; p 510.

(6) Fick, A. On liquid diffusion. Journal of Membrane Science 1995, 100, 33-38.

(7) Liu, X.; Schnell, S. K.; Simon, J. M.; Krüger, P.; Bedeaux, D.; Kjelstrup, S.; Bardow, A.; Vlugt, T. J. H. Diffusion Coefficients from Molecular Dynamics Simulations in Binary and Ternary Mixtures. International Journal of Thermophysics 2013, 34, 1169-1196.

(8) Taylor, R.; Krishna, R. Multicomponent Mass Transfer, first edit ed.; John Wiley \& Sons, INC.: New York, 1954; p 579.

(9) Maxwell, J. C. On the Dynamical Theory of Gases. Philosophical Transactions of the Royal Society of London 1867, 157, 49-88.

(10) Stefan, J. Über das Gleichgewicht und die Bewegung, insbesondere die Diffusion von Gasgemengen. Sitzber. Akad. Wiss. Wien 1871, 63, 63-124. 
(11) Bird, R. B.; Stewart, W. E.; Lightfoot, E. N. Transport Phenomena, second edi ed.; John Wiley \& Sons, INC.: New York, 2001; p 780.

(12) Cussler, E. L. Diffusion Mass Transfer in Fluid Systems, third edit ed.; Cambridge University Press: New York, 2009.

(13) Curtiss, C. F.; Byron Bird, R. Multicomponent diffusion. Industrial and Engineering Chemistry Research 1999, 38, 2515-2522.

(14) Krishna, R.; Van Baten, J. M. The darken relation for multicomponent diffusion in liquid mixtures of linear alkanes: An investigation using Molecular Dynamics (MD) simulations. Industrial and Engineering Chemistry Research 2005, 44, 6939-6947.

(15) Krishna, R.; Wesselingh, J. A. The Maxwell-Stefan approach to mass transfer. Chemical Engineering Science 1997, 52, 861-911.

(16) Hines, R. N.; Maddox, A. L. Mass transfer: fundamentals and applications; Prentice Hall: New Jesery, 1985; p 600.

(17) Liu, X.; Martín-Calvo, A.; McGarrity, E.; Schnell, S. K.; Calero, S.; Simon, J. M.; Bedeaux, D.; Kjelstrup, S.; Bardow, A.; Vlugt, T. J. H. Fick diffusion coefficients in ternary liquid systems from equilibrium molecular dynamics simulations. Industrial and Engineering Chemistry Research 2012, 51, 10247-10258.

(18) Wheeler, D. R.; Newman, J. Molecular Dynamics Simulations of Multicomponent Diffusion in Liquid Electrolytes. The Journal of Physical Chemistry B 2004, 108, 1836218367.

(19) Yang, H.; Zhang, J.; Müller-Plathe, F.; Yang, Y. A reverse nonequilibrium molecular dynamics method for calculating the mutual diffusion coefficient for binary fluids. Chemical Engineering Science 2015, 130, 1-7. 
(20) Yang, H.; Zhang, J.; Müller-Plathe, F. Extending reverse nonequilibrium molecular dynamics to the calculation of mutual diffusion coefficients in molecular fluid mixtures. Molecular Simulation 2016, 42, 1379-1384.

(21) Zabala, D.; Nieto-Draghi, C.; de Hemptinne, J. C.; Ramos, A. L. Diffusion Coefficients in $\mathrm{CO} 2 / \mathrm{n}$-Alkane Binary Liquid Mixtures by Molecular Simulation. The Journal of Physical Chemistry B 2008, 112, 16610-16618.

(22) Nichols, J. W.; Wheeler, D. R. Fourier Correlation Method for Simulating Mutual Diffusion Coefficients in Condensed Systems at Equilibrium. Industrial and Engineering Chemistry Research 2015, 54, 12156-12164.

(23) Guevara-Carrion, G.; Janzen, T.; Muñoz-Muñoz, Y. M.; Vrabec, J. Mutual diffusion of binary liquid mixtures containing methanol, ethanol, acetone, benzene, cyclohexane, toluene, and carbon tetrachloride. Journal of Chemical Physics 2016, 144.

(24) Jacucci, G.; McDonald, I. R. Structure and diffusion in mixtures of rare-gas liquids. Physica A: Statistical Mechanics and its Applications 1975, 80, 607-625.

(25) Onsager, L. Reciprocal Relations in Irreversible Processes. I. Physical Review 1931, $37,405-426$.

(26) Schnell, S. K.; Bedeaux, D.; Kjelstrup, S.; Dawass, N.; Vlugt, T. J. H.; Krüger, P.; Simon, J. M. Finite-size effects of Kirkwood-Buff integrals from molecular simulations. Molecular Simulation 2017, 44, 599-612.

(27) Krüger, P.; Vlugt, T. J. Size and shape dependence of finite-volume Kirkwood-Buff integrals. Physical Review E 2018, 97, 1-5.

(28) Dawass, N.; Krüger, P.; Schnell, S. K.; Simon, J. M.; Vlugt, T. J. Kirkwood-Buff integrals from molecular simulation. Fluid Phase Equilibria 2019, 486, 21-36. 
(29) Fingerhut, R.; Vrabec, J. Kirkwood-Buff integration: A promising route to entropic properties? Fluid Phase Equilibria 2019, 485, 270-281.

(30) Nichols, J. W.; Moore, S. G.; Wheeler, D. R. Improved implementation of KirkwoodBuff solution theory in periodic molecular simulations. Physical Review E - Statistical, Nonlinear, and Soft Matter Physics 2009, 80, 1-10.

(31) Hansen, J.-P.; McDonald, I. R. Theory of simple liquids: with applications to soft matter; Academic Press: Oxford, England, 2013; OCLC: 1048428149.

(32) Rogers, D. M. Extension of Kirkwood-Buff theory to the canonical ensemble. Journal of Chemical Physics 2018, 148, 8-11.

(33) Jorgensen, W. L.; Madura, J. D.; Swenson, C. J. Optimized Intermolecular Potential Functions for Liquid Hydrocarbons. Journal of the American Chemical Society 1984, $106,6638-6646$.

(34) Higashi, H.; Iwai, Y.; Uchida, H.; Arai, Y. Diffusion coefficients of aromatic compounds in supercritical carbon dioxide using molecular dynamics simulation. Journal of Supercritical Fluids 1998, 13, 93-97.

(35) Aimoli, C. G.; Maginn, E. J.; Abreu, C. R. A. Force field comparison and thermodynamic property calculation of supercritical CO2and CH4using molecular dynamics simulations. Fluid Phase Equilibria 2014, 368, 80-90.

(36) Aimoli, C. G.; Maginn, E. J.; Abreu, C. R. A. Transport properties of carbon dioxide and methane from molecular dynamics simulations. The Journal of Chemical Physics 2014, 141, 134101.

(37) Abreu, C. R. A. Playmol. https://github.com/atoms-ufrj/playmol, 2018.

(38) Plimpton, S. Fast Parallel Algorithms for Short - Range Molecular Dynamics. Journal of Computational Physics 1995, 117, 1-19. 
(39) Tuckerman, M. E.; Alejandre, J.; López-Rendón, R.; Jochim, A. L.; Martyna, G. J. A Liouville-operator derived measure-preserving integrator for molecular dynamics simulations in the isothermal-isobaric ensemble. Journal of Physics A: Mathematical and General 2006, 39, 5629-5651.

(40) Martyna, G. J.; Klein, M. L.; Tuckerman, M. Nosé - Hoover chains : The canonical ensemble via continuous dynamics Nose-Hoover chains : The canonical ensemble via continuous dynamics. Journal of Chemical Physics 1992, 2635, 2635-2643.

(41) Martyna, G. J.; Tobias, D. J.; Klein, M. L. Constant pressure molecular dynamics algorithms. The Journal of Chemical Physics 1994, 101, 4177-4189.

(42) Dubbeldam, D.; Ford, D. C.; Ellis, D. E.; Snurr, R. Q. A new perspective on the order-n algorithm for computing correlation functions. Molecular Simulation 2009, 35, $1084-1097$.

(43) March, N. H.; Tosi, M. P. Atomic Dynamics in Liquids, first edit ed.; The MacMillan Press LTD: London, 1976.

(44) Segtovich, I. S. V. Estimation. https://github.com/iurisegtovich/estimation, 2019.

(45) Schwaab, M.; Biscaia, E. C.; Monteiro, J. L.; Pinto, J. C. Nonlinear parameter estimation through particle swarm optimization. Chemical Engineering Science 2008, 63, $1542-1552$.

(46) Bard, Y. Nonlinear Parameter Estimation; Academic Press: New York, 1974; p 341.

(47) Kunz, O.; Wagner, W. The GERG-2008 wide-range equation of state for natural gases and other mixtures: An expansion of GERG-2004. Journal of Chemical and Engineering Data 2012, 57, 3032-3091. 
(48) Lemmon, E.; Huber, M.; McLinden, M. Reference Fluid Thermodynamic and Transport Properties -REFPROP, Version 7.0. - User's Guide. 2003.

(49) Krüger, P.; Schnell, S. K.; Bedeaux, D.; Kjelstrup, S.; Vlugt, T. J.; Simon, J. M. Kirkwood-Buff integrals for finite volumes. Journal of Physical Chemistry Letters 2013, 4, $235-238$.

(50) Cadogan, S. P.; Mistry, B.; Wong, Y.; Maitland, G. C.; Trusler, J. P. M. Diffusion Coefficients of Carbon Dioxide in Eight Hydrocarbon Liquids at Temperatures between (298.15 and 423.15) K at Pressures up to $69 \mathrm{MPa}$. Journal of Chemical and Engineering Data 2016, 61, 3922-3932.

(51) Wang, L. S.; Lang, Z. X.; Guo, T. M. Measurement and correlation of the diffusion coefficients of carbon dioxide in liquid hydrocarbons under elevated pressure. Fluid Phase Equilibria 1996, 117, 364-372.

(52) Glaser, J.; Hallatschek, O.; Kroy, K. Dynamic structure factor of a stiff polymer in a glassy solution. European Physical Journal E 2008, 26, 123-136.

(53) Jamali, S. H.; Wolff, L.; Becker, T. M.; Bardow, A.; Vlugt, T. J.; Moultos, O. A. FiniteSize Effects of Binary Mutual Diffusion Coefficients from Molecular Dynamics. Journal of Chemical Theory and Computation 2018, 14, 2667-2677.

(54) Higgoda, U. A.; Kankanamge, C. J.; Hellmann, R.; Koller, T. M.; Fröba, A. P. Fick diffusion coefficients of binary fluid mixtures consisting of methane, carbon dioxide, and propane via molecular dynamics simulations based on simplified pair-specific ab initio-derived force fields. Fluid Phase Equilibria 2019, 112257.

(55) Yeh, I. C.; Hummer, G. System-size dependence of diffusion coefficients and viscosities from molecular dynamics simulations with periodic boundary conditions. Journal of Physical Chemistry B 2004, 108, 15873-15879. 
(56) Hasimoto, H. On the periodic fundamental solutions of the Stokes equations and their application to viscous. J. Fluid Mech. 1958, 5, 317-328. 


\section{Table of Content (Graphical Abstract)}

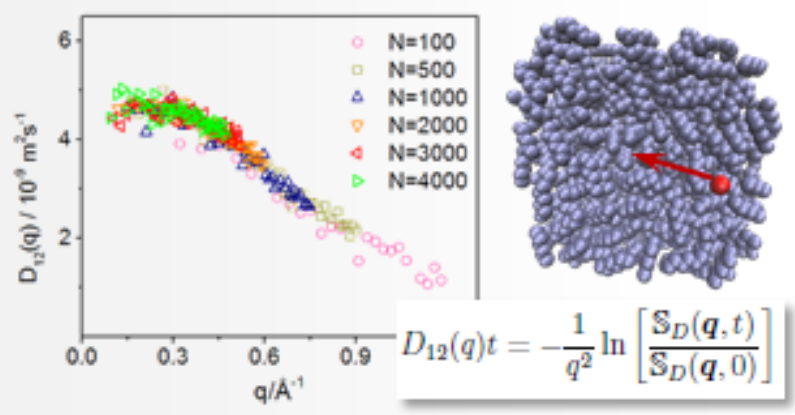

Figure 11: For Table of Contents Only 


\section{Supplementary Material}

Table S1: Parameters of OPLS-UA ${ }^{33}$ and Higashi ${ }^{34}$ force fields used in LAMMPS

\begin{tabular}{|c|c|c|c|c|}
\hline atom_style & full & & & \\
\hline & $\epsilon / k_{c a l} . \mathrm{mol}^{-1}$ & $\sigma / \AA$ & Mass/Da & Charge \\
\hline $\mathrm{CO}_{2}$ & 0.4692 & 3.9050 & 44.010 & 0.000 \\
\hline $\mathrm{CH}_{3}^{-}$ & 0.1750 & 3.9050 & 15.035 & 0.000 \\
\hline $\mathrm{CH}_{2}$ & 0.1180 & 3.9050 & 14.027 & 0.000 \\
\hline bond_style & harmonic & & & \\
\hline & $K_{r} / k_{c a l} \cdot \mathrm{mol}^{-1} \AA^{-2}$ & $r_{e q} / \AA$ & & \\
\hline $\mathrm{CH}_{3}-\mathrm{CH}_{2}$ & 260.0 & 1.526 & & \\
\hline $\mathrm{CH}_{2}-\mathrm{CH}_{2}$ & 260.0 & 1.526 & & \\
\hline angle_style & harmonic & & & \\
\hline & $K_{\theta} / k_{c a l . m o l}{ }^{-1} \mathrm{rad}^{-2}$ & $\theta_{e q} / d e g$ & & \\
\hline $\mathrm{CH}_{3}-\mathrm{CH}_{2}-\mathrm{CH}_{2}$ & 63.0 & 112.4 & & \\
\hline $\mathrm{CH}_{2}-\mathrm{CH}_{2}-\mathrm{CH}_{2}$ & 63.0 & 112.4 & & \\
\hline dihedral_style & opls & & & \\
\hline & $K_{1} / k_{c a l . m o l}-1$ & $K_{2} /$ kcal.mol $^{-1}$ & $K_{3} /$ kcal.mol $^{-1}$ & $K_{4} / k_{c a l . m o l}-1$ \\
\hline $\mathrm{CH}_{3}-\mathrm{CH}_{2}-\mathrm{CH}_{2}-\mathrm{CH}_{2}$ & 1.4110 & -0.2710 & 3.1450 & 0.0000 \\
\hline $\mathrm{CH}_{2}-\mathrm{CH}_{2}-\mathrm{CH}_{2}-\mathrm{CH}_{2}$ & 1.4110 & -0.2710 & 3.1450 & 0.0000 \\
\hline pair_style & $\mathrm{lj} /$ cut & & & \\
\hline $\begin{array}{l}\text { Combining rules } \\
\text { special_bonds }\end{array}$ & $\begin{array}{l}\text { Geometric } \\
\text { lj } 0.00 .00 .0\end{array}$ & & & \\
\hline
\end{tabular}



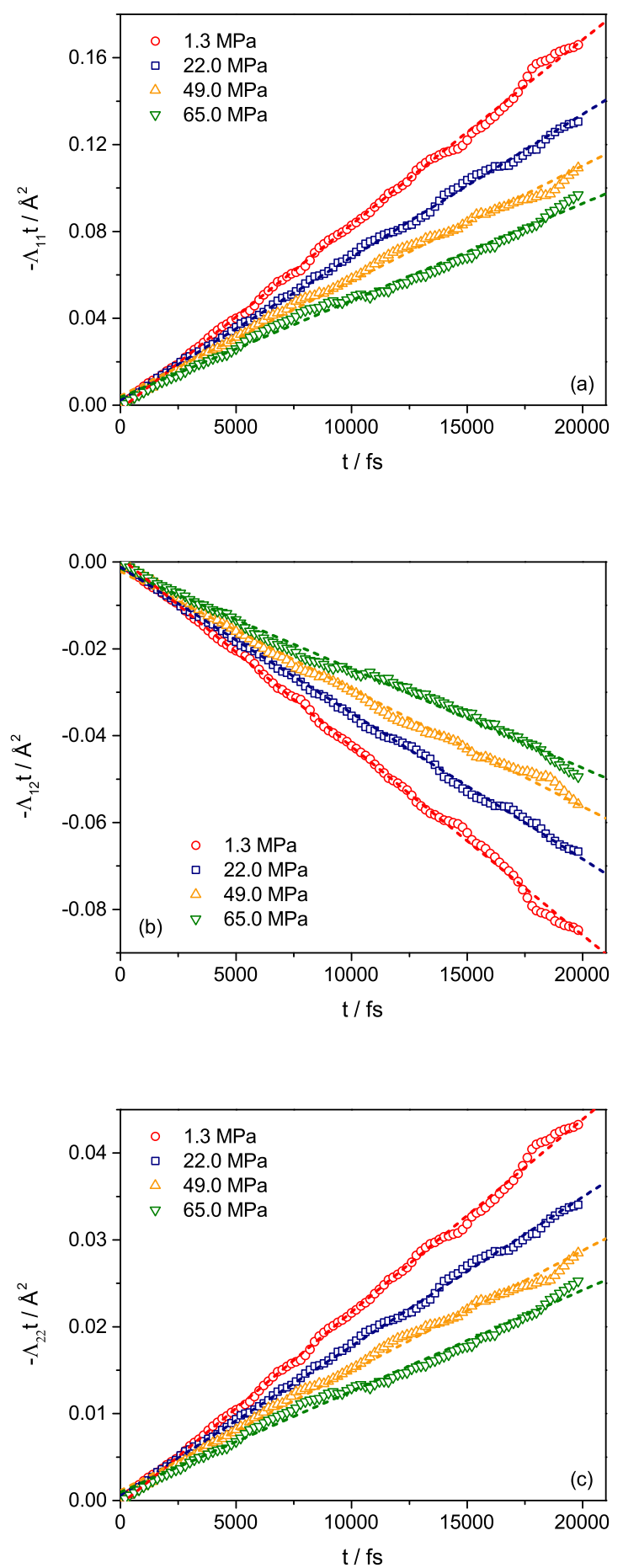

Figure S1: Results of the Onsager coefficients (a) $\Lambda_{11}$, (b) $\Lambda_{12}$, and (c) $\Lambda_{22}$ for $\mathrm{CO}_{2}+$ n-hexane mixtures at infinity dilution of $\mathrm{CO}_{2}$, at $290.0 \mathrm{~K}$ and under different pressures. The symbols represents the simulation results over time, and the dashed lines are the linear regressions performed for each case. 
Table S2: Density and thermodynamic factor results for $\mathrm{CO}_{2}+\mathrm{n}$-hexane mixtures at infinity dilution of $\mathrm{CO}_{2}$, under different conditions of temperature and pressure.

\begin{tabular}{|c|c|c|c|c|c|}
\hline \multirow{2}{*}{$\mathbf{T} / \mathbf{K}$} & \multirow{2}{*}{$\mathrm{P} / \mathrm{MPa}$} & \multicolumn{3}{|c|}{$\rho / k g \cdot m^{-3}$} & \multirow{2}{*}{$\Gamma$} \\
\hline & & $\mathrm{MD}$ & GERG-2008 & Dev.(\%) & \\
\hline \multirow{4}{*}{298.0} & 1.3 & $681.63 \pm 3.44$ & 664.27 & $2.61 \%$ & $1.00 \pm 0.01$ \\
\hline & 22.0 & $698.03 \pm 3.19$ & 678.76 & $2.84 \%$ & $1.01 \pm 0.01$ \\
\hline & 49.0 & $715.85 \pm 2.99$ & 698.56 & $2.47 \%$ & $1.02 \pm 0.01$ \\
\hline & 65.0 & $724.89 \pm 2.82$ & 708.39 & $2.33 \%$ & $1.06 \pm 0.01$ \\
\hline \multirow{4}{*}{323.0} & 1.2 & $662.54 \pm 3.74$ & 643.17 & $3.01 \%$ & $0.99 \pm 0.01$ \\
\hline & 22.0 & $681.52 \pm 3.42$ & 659.32 & $3.37 \%$ & $1.00 \pm 0.01$ \\
\hline & 50.0 & $701.64 \pm 3.15$ & 682.50 & $2.80 \%$ & $0.99 \pm 0.01$ \\
\hline & 66.0 & $711.31 \pm 3.03$ & 693.31 & $2.60 \%$ & $1.05 \pm 0.01$ \\
\hline \multirow{4}{*}{373.0} & 1.2 & $621.12 \pm 4.65$ & 598.13 & $3.84 \%$ & $0.98 \pm 0.01$ \\
\hline & 22.0 & $647.03 \pm 3.96$ & 619.85 & $4.38 \%$ & $1.03 \pm 0.01$ \\
\hline & 49.0 & $671.53 \pm 3.55$ & 648.77 & $3.51 \%$ & $1.00 \pm 0.01$ \\
\hline & 64.0 & $682.51 \pm 3.41$ & 661.27 & $3.21 \%$ & $0.98 \pm 0.01$ \\
\hline
\end{tabular}


Table S3: Density and thermodynamic factor results for $\mathrm{CO}_{2}+\mathrm{n}$-octane mixtures at infinity dilution of $\mathrm{CO}_{2}$, under different conditions of temperature and pressure.

\begin{tabular}{|c|c|c|c|c|c|}
\hline \multirow{2}{*}{$\mathbf{T} / \mathbf{K}$} & \multirow{2}{*}{$\mathbf{P} / \mathbf{M P a}$} & \multicolumn{3}{|c|}{$\rho / k g \cdot m^{-3}$} & \multirow{2}{*}{$\Gamma$} \\
\hline & & $\mathrm{MD}$ & GERG-2008 & Dev.(\%) & \\
\hline \multirow{5}{*}{298.0} & 1.0 & $738.72 \pm 2.89$ & 700.35 & $5.48 \%$ & $1.02 \pm 0.01$ \\
\hline & 10.0 & $745.52 \pm 2.84$ & 708.05 & $5.29 \%$ & $0.97 \pm 0.01$ \\
\hline & 31.0 & $758.74 \pm 2.69$ & 723.41 & $4.88 \%$ & $1.06 \pm 0.01$ \\
\hline & 50.0 & $767.69 \pm 2.53$ & 735.16 & $4.42 \%$ & $1.00 \pm 0.01$ \\
\hline & 68.0 & $775.32 \pm 2.47$ & 744.98 & $4.07 \%$ & $1.06 \pm 0.01$ \\
\hline \multirow{5}{*}{323.0} & 1.0 & $721.39 \pm 3.13$ & 679.88 & $6.11 \%$ & $1.04 \pm 0.01$ \\
\hline & 10.0 & $729.40 \pm 3.03$ & 688.94 & $5.87 \%$ & $1.02 \pm 0.01$ \\
\hline & 30.0 & $743.38 \pm 2.86$ & 705.74 & $5.33 \%$ & $1.01 \pm 0.01$ \\
\hline & 51.0 & $754.48 \pm 2.69$ & 720.20 & $4.76 \%$ & $0.97 \pm 0.01$ \\
\hline & 68.0 & $763.17 \pm 2.63$ & 730.34 & $4.49 \%$ & $1.00 \pm 0.01$ \\
\hline
\end{tabular}


Table S4: Density and thermodynamic factor results for $\mathrm{CO}_{2}+\mathrm{n}$-octane mixtures at concentrations, temperatures, and pressures.

\begin{tabular}{|c|c|c|c|c|c|c|}
\hline \multirow{2}{*}{$\mathbf{T} / \mathbf{K}$} & \multirow{2}{*}{$x_{\mathrm{CO}_{2}}$} & \multirow{2}{*}{$\mathrm{P} / \mathrm{MPa}$} & \multicolumn{3}{|c|}{$\rho / k g \cdot m^{-3}$} & \multirow{2}{*}{$\Gamma$} \\
\hline & & & MD & GERG-2008 & Dev.(\%) & \\
\hline \multirow{5}{*}{290.0} & 0.2427 & 1.265 & $763.55 \pm 3.43$ & 730.67 & $4.50 \%$ & $1.03 \pm 0.01$ \\
\hline & 0.3324 & 1.843 & $772.85 \pm 3.64$ & 742.09 & $4.15 \%$ & $1.15 \pm 0.01$ \\
\hline & 0.4124 & 2.358 & $782.87 \pm 4.03$ & 753.32 & $3.92 \%$ & $1.12 \pm 0.01$ \\
\hline & 0.5216 & 3.061 & $798.46 \pm 4.61$ & 770.49 & $3.63 \%$ & $1.08 \pm 0.01$ \\
\hline & 0.6167 & 3.673 & $813.91 \pm 5.32$ & 787.47 & $3.36 \%$ & $1.09 \pm 0.01$ \\
\hline \multirow{5}{*}{311.0} & 0.1344 & 0.747 & $738.25 \pm 3.34$ & 699.49 & $5.54 \%$ & $1.07 \pm 0.01$ \\
\hline & 0.2384 & 1.667 & $747.35 \pm 3.59$ & 709.69 & $5.31 \%$ & $1.04 \pm 0.01$ \\
\hline & 0.2499 & 1.769 & $748.47 \pm 3.70$ & 710.88 & $5.29 \%$ & $1.08 \pm 0.01$ \\
\hline & 0.2814 & 2.048 & $751.40 \pm 3.72$ & 714.18 & $5.21 \%$ & $1.08 \pm 0.01$ \\
\hline & 0.4007 & 3.103 & $764.34 \pm 4.30$ & 727.49 & $5.07 \%$ & $1.08 \pm 0.01$ \\
\hline
\end{tabular}


Table S5: Static structure factor values at the thermodynamic limit for $\mathrm{CO}_{2}+\mathrm{n}$-hexane mixtures at infinity dilution of $\mathrm{CO}_{2}$, under different conditions of temperature and pressure.

\begin{tabular}{cc|c|c|c}
\hline $\mathbf{T} / \mathbf{K}$ & $\mathbf{P} / \mathbf{M P a}$ & $S_{11}(0) / 10^{-3}$ & $S_{12}(0) / 10^{-3}$ & $S_{22}(0) / 10^{-3}$ \\
\hline & 1.3 & $10.06 \pm 0.02$ & $-3.45 \pm 0.01$ & $24.26 \pm 0.11$ \\
& 22.0 & $9.93 \pm 0.02$ & $-3.40 \pm 0.01$ & $19.82 \pm 0.07$ \\
& 49.0 & $9.85 \pm 0.02$ & $-3.19 \pm 0.01$ & $16.24 \pm 0.05$ \\
& 65.0 & $9.45 \pm 0.02$ & $-3.21 \pm 0.01$ & $14.90 \pm 0.04$ \\
\hline \multirow{3}{*}{323.0} & 1.2 & $10.12 \pm 0.02$ & $-3.51 \pm 0.01$ & $30.40 \pm 0.17$ \\
& 22.0 & $10.06 \pm 0.02$ & $-3.62 \pm 0.01$ & $24.19 \pm 0.11$ \\
& 50.0 & $10.12 \pm 0.02$ & $-3.44 \pm 0.01$ & $19.33 \pm 0.07$ \\
& 66.0 & $9.55 \pm 0.02$ & $-3.17 \pm 0.01$ & $17.24 \pm 0.06$ \\
\hline \multirow{3}{*}{373.0} & 1.2 & $10.24 \pm 0.02$ & $-3.81 \pm 0.01$ & $50.02 \pm 0.47$ \\
& 22.0 & $9.73 \pm 0.02$ & $-3.61 \pm 0.01$ & $35.43 \pm 0.23$ \\
& 49.0 & $10.05 \pm 0.02$ & $-3.39 \pm 0.01$ & $26.58 \pm 0.13$ \\
& 64.0 & $10.26 \pm 0.02$ & $-3.53 \pm 0.01$ & $23.79 \pm 0.11$ \\
\hline
\end{tabular}


Table S6: Static structure factor values at the thermodynamic limit for $\mathrm{CO}_{2}+$ n-octane mixtures at infinity dilution of $\mathrm{CO}_{2}$, under different conditions of temperature and pressure.

\begin{tabular}{cc|c|c|c}
\hline $\mathbf{T} / \mathbf{K}$ & $\mathbf{P} / \mathbf{M P a}$ & $S_{11}(0) / 10^{-3}$ & $S_{12}(0) / 10^{-3}$ & $S_{22}(0) / 10^{-3}$ \\
\hline & 1.0 & $9.87 \pm 0.02$ & $-2.65 \pm 0.01$ & $14.68 \pm 0.04$ \\
& 10.0 & $10.33 \pm 0.02$ & $-2.72 \pm 0.01$ & $13.77 \pm 0.04$ \\
298.0 & 31.0 & $9.51 \pm 0.02$ & $-2.55 \pm 0.01$ & $11.93 \pm 0.03$ \\
& 50.0 & $10.03 \pm 0.02$ & $-2.66 \pm 0.01$ & $10.95 \pm 0.02$ \\
& 68.0 & $9.47 \pm 0.02$ & $-2.52 \pm 0.01$ & $10.18 \pm 0.02$ \\
\hline & 1.0 & $9.63 \pm 0.02$ & $-2.76 \pm 0.01$ & $17.86 \pm 0.06$ \\
323.0 & 10.0 & $9.87 \pm 0.02$ & $-2.74 \pm 0.01$ & $16.22 \pm 0.05$ \\
& 30.0 & $9.93 \pm 0.02$ & $-2.70 \pm 0.01$ & $14.04 \pm 0.04$ \\
& 51.0 & $10.34 \pm 0.02$ & $-2.84 \pm 0.01$ & $12.56 \pm 0.03$ \\
& 68.0 & $10.09 \pm 0.02$ & $-2.71 \pm 0.01$ & $11.50 \pm 0.02$ \\
\hline
\end{tabular}


Table S7: Static structure factor values at the thermodynamic limit for $\mathrm{CO}_{2}+$ n-octane mixtures at different concentrations, temperatures, and pressures.

\begin{tabular}{ccc|c|c|c}
\hline $\mathbf{T} / \mathbf{K}$ & $x_{C O_{2}}$ & $\mathbf{P} / \mathbf{M P a}$ & $S_{11}(0) / 10^{-3}$ & $S_{12}(0) / 10^{-3}$ & $S_{22}(0) / 10^{-3}$ \\
\hline & 0.2427 & 1.265 & $263.10 \pm 12.93$ & $-71.47 \pm 0.95$ & $31.15 \pm 0.18$ \\
& 0.3324 & 1.843 & $332.23 \pm 20.62$ & $-92.21 \pm 1.59$ & $37.08 \pm 0.26$ \\
290.0 & 0.4124 & 2.358 & $435.34 \pm 35.40$ & $-121.74 \pm 2.77$ & $45.33 \pm 0.38$ \\
& 0.5216 & 3.061 & $585.38 \pm 64.00$ & $-164.50 \pm 5.05$ & $57.46 \pm 0.62$ \\
& 0.6167 & 3.673 & $677.66 \pm 85.77$ & $-192.85 \pm 6.95$ & $66.82 \pm 0.83$ \\
\hline & 0.1344 & 0.747 & $133.23 \pm 3.32$ & $-36.79 \pm 0.25$ & $25.09 \pm 0.12$ \\
& 0.2384 & 1.667 & $252.22 \pm 11.88$ & $-70.58 \pm 0.93$ & $33.96 \pm 0.22$ \\
311.0 & 0.2499 & 1.769 & $257.76 \pm 12.41$ & $-72.38 \pm 0.98$ & $34.33 \pm 0.22$ \\
& 0.2814 & 2.048 & $293.42 \pm 16.08$ & $-82.50 \pm 1.27$ & $37.08 \pm 0.26$ \\
& 0.4007 & 3.103 & $432.43 \pm 34.93$ & $-122.06 \pm 2.78$ & $48.09 \pm 0.43$ \\
\hline
\end{tabular}

